\title{
On the Łojasiewicz-Simon gradient inequality on submanifolds
}

\author{
Fabian Rupp*
}

July 21,2020

\begin{abstract}
We provide sufficient conditions for the Eojasiewicz-Simon gradient inequality to hold on a submanifold of a Banach space and discuss the optimality of our assumptions. Our result provides a tool to study asymptotic properties of quasilinear parabolic equations with (nonlinear) constraints.
\end{abstract}

Keywords: Łojasiewicz-Simon gradient inequality, constrained gradient flows, mean curvature flow with isoperimetric constraint, constrained Allen-Cahn equation.

MSC(2010): 26D10 (primary), 46T05, 37C10 (secondary).

\section{Introduction}

In real algebraic geometry, the Eojasiewicz inequality is a remarkable result describing the particular behavior of an analytic function near a critical point.

Theorem 1.1 (Łojasiewicz inequality, [27, Théorème 4]). Let $U \subset \mathbb{R}^{n}$ be open. If $\mathcal{E} \in \mathcal{C}^{\omega}(U ; \mathbb{R})$ and $\bar{u} \in U$ satisfies $\nabla \mathcal{E}(\bar{u})=0$, then there exist $C, \sigma>0$ and $\theta \in\left(0, \frac{1}{2}\right]$ such that for all $\|u-\bar{u}\| \leq \sigma$, we have

$$
|\mathcal{E}(u)-\mathcal{E}(\bar{u})|^{1-\theta} \leq C\|\nabla \mathcal{E}(u)\| .
$$

Throughout this article, we write $\mathcal{C}^{\omega}(U ; X)$ for the set of real analytic functions from an open set $U$ of a Banach space $V$ into another Banach space $X$. All vector spaces are understood to be over the field of real numbers $\mathbb{R}$. The space of bounded linear operators between two normed spaces $X$ and $Y$ is denoted by $\mathcal{L}(X, Y)$ and we write $X^{*}:=\mathcal{L}(X, \mathbb{R})$ for the continuous dual of $X$.

In $\mathbb{R}^{n}$, inequality (11) was discovered and proven by $\mathrm{S}$. Lojasiewicz in his famous works on semianalytic and subanalytic sets, [27, 28]. Since then, Theorem 1.1] has been used as

\footnotetext{
*Institute of Analysis, Ulm University, Helmholtzstraße 18, 89081 Ulm, Germany. fabian.rupp@uni-ulm.de.
} 
a celebrated tool to prove convergence results for the gradient flow of analytic energies on finite-dimensional spaces (see [29]). The pioneering work of L. Simon in [33] extended inequality (1) to certain energy functions on infinite-dimensional function spaces using Lyapunov-Schmidt reduction and, in honor of his significant contributions, the inequality is nowadays often called Eojasiewicz-Simon gradient inequality. In more recent work by Kurdyka [22], Łojasiewicz's convergence result has been extended to a larger class of functions via the Kurdyka-Lojasiewicz inequality. Over the last decades, gradient inequalities like (11) have been extensively studied in various situations to analyze the long time behavior of gradient flows, see for instance [12, 13, 15, 18, 32]. In [20, 21], this is also done for second order evolution equations. Loosely speaking, whenever an energy $\mathcal{E}$ satisfies a Łojasiewicz-Simon gradient inequality at a critical point $\bar{u}=\lim _{n \rightarrow \infty} u\left(t_{n}\right)$, where $t_{n} \rightarrow \infty$ and $u=u(t)$ is a precompact solution to the associated gradient flows

$$
\begin{cases}\partial_{t} u & =-\nabla \mathcal{E}(u), \quad t>0 \\ u(0) & =u_{0}\end{cases}
$$

we may conclude that $u$ converges with $\lim _{t \rightarrow \infty} u(t)=\bar{u}$. Numerical applications of this phenomenon have been considered for instance in [2, 6].

Hence, it is a question of great interest, whether a given energy function satisfies a Łojasiewicz-Simon gradient inequality. It can be shown that in the infinite-dimensional case, mere analyticity of the energy is not enough, see for instance [19, Theorem 2.1, Proposition 3.5]. On the other hand, very general conditions which are sufficient for the gradient inequality to hold are presented in [10.

For most of the applications, one usually checks that the following conditions are satisfied, see [12, 13, 14, 25].

Theorem 1.2 (Consequence of [10, Corollary 3.11]). Let $V$ be a Banach space, $U \subset V$ an open set, $\mathcal{E} \in \mathcal{C}^{\omega}(U ; \mathbb{R})$ and $\bar{u} \in U$ a critical point of $\mathcal{E}$. Suppose that

(i) there exists a Banach space $Z$ such that $V \hookrightarrow Z$ densely,

(ii) $\mathcal{E}^{\prime} \in \mathcal{C}^{\omega}\left(U ; Z^{*}\right)$,

(iii) the second derivative $\mathcal{E}^{\prime \prime}(\bar{u}): V \rightarrow Z^{*}$ is Fredholm of index zero.

Then, there exist $C, \sigma>0, \theta \in\left(0, \frac{1}{2}\right]$ such that for all $u \in U$ with $\|u-\bar{u}\|_{V} \leq \sigma$, we have

$$
|\mathcal{E}(u)-\mathcal{E}(\bar{u})|^{1-\theta} \leq C\left\|\mathcal{E}^{\prime}(u)\right\|_{Z^{*}} .
$$

Remark 1.3. Note that by assumption (i) in Theorem 1.2 we have $V \hookrightarrow Z$, so $Z^{*}$ can be identified with a subset of $V^{*}$. Condition (ii) requires that for all $u \in U$ the functional $\mathcal{E}^{\prime}(u)$ which is in general only in $V^{*}$ is in fact in $Z^{*}$ and the map $\mathcal{E}^{\prime}: U \rightarrow Z^{*}$ is analytic.

Although Theorem 1.2 describes a slightly less general situation than in [10], in most applications its conditions are relatively easy to check and suffice to prove the ŁojasiewiczSimon gradient inequality. The details on how to deduce Theorem 1.2 from [10] are given in Appendix A. 
To prove a suitable version of Theorem 1.1 on a finite-dimensional manifold $\mathcal{M}$ is quite straightforward if $\mathcal{M}$ and $\mathcal{E}$ are analytic, by simply choosing local coordinates and applying Theorem 1.1. In [23], this is used to study gradient-like dynamical systems via the Kurdyka-Łojasiewicz inequality. The infinite-dimensional setting is more complicated. Our main result is to extend Theorem 1.2 to a constrained energy function $\left.\mathcal{E}\right|_{\mathcal{M}}$ on a submanifold $\mathcal{M}$ of a Banach space $V$, and to refine the estimate by projecting the derivative onto the cotangent space of $\mathcal{M}$. In [25], a special case has been studied and a Łojasiewicz-Simon gradient inequality is proven for the Canham-Helfrich energy on the submanifold of closed embedded surfaces with fixed area and volume, see [25, Theorem 1.4]. In the following theorem, we give very general sufficient conditions for the Łojasiewciz-Simon gradient inequality to hold on an infinite-dimensional submanifold in the abstract setting of an energy on a Banach space. In Section 5, we will consider the easier case where the ambient space is a Hilbert space. However, as we shall explain in detail in Remark 1.7 below, in order to avoid issues with analyticity, it is sometimes necessary to work in Banach spaces, cf. also Section 7.1. Our main result is the following

Theorem 1.4. Let $V$ be a Banach space, $U \subset V$ an open set, $m \in \mathbb{N}$ and $\mathcal{E}: U \rightarrow \mathbb{R}$, $\mathcal{G}: U \rightarrow \mathbb{R}^{m}$ be analytic. Let $\bar{u} \in U$ and suppose that

(i) there exists a Banach space $Y$ such that $V \hookrightarrow Y$ densely,

(ii) $\mathcal{E}^{\prime} \in \mathcal{C}^{\omega}\left(U ; Y^{*}\right)$,

(iii) the second derivative $\mathcal{E}^{\prime \prime}(\bar{u}): V \rightarrow Y^{*}$ is Fredholm of index zero,

(iv) for any $u \in U$, the linear operator $\mathcal{G}^{\prime}(u) \in \mathcal{L}\left(V, \mathbb{R}^{m}\right)$ extends to $\overline{\mathcal{G}^{\prime}(u)} \in \mathcal{L}\left(Y, \mathbb{R}^{m}\right)$ and the map $\overline{\mathcal{G}^{\prime}}: U \rightarrow \mathcal{L}\left(Y, \mathbb{R}^{m}\right), u \mapsto \overline{\mathcal{G}^{\prime}(u)}$ is analytic,

(v) the Fréchet derivative $\left(\overline{\mathcal{G}^{\prime}}\right)^{\prime}(\bar{u}): V \rightarrow \mathcal{L}\left(Y, \mathbb{R}^{m}\right)$ is compact,

(vi) $\mathcal{G}(\bar{u})=0$ and $\mathcal{G}^{\prime}(\bar{u}): V \rightarrow \mathbb{R}^{m}$ is surjective.

Then, $\mathcal{M}:=\{u \in U \mid \mathcal{G}(u)=0\}$ is locally an analytic submanifold of $V$ of codimension $m$ near $\bar{u}$.

If $\bar{u}$ is a critical point of $\left.\mathcal{E}\right|_{\mathcal{M}}$, then the restriction satisfies a refined Lojasiewicz-Simon gradient inequality at $\bar{u}$, i.e. there exist $C, \sigma>0$ and $\theta \in\left(0, \frac{1}{2}\right]$ such that for any $u \in \mathcal{M}$ with $\|u-\bar{u}\|_{V} \leq \sigma$, we have

$$
|\mathcal{E}(u)-\mathcal{E}(\bar{u})|^{1-\theta} \leq C\left\|\mathcal{E}^{\prime}(u)\right\|_{\overline{\mathcal{T}}_{u \mathcal{M}}} .
$$

Here, ${\overline{\mathcal{T}_{u} \mathcal{M}}}^{*}$ is the dual of the closure $\overline{\mathcal{T}_{u} \mathcal{M}}:={\overline{\mathcal{T}_{u} \mathcal{M}}}^{\|\cdot\|_{Y}} \subset Y$ of the tangent space $\mathcal{T}_{u} \mathcal{M}$.

Remark 1.5. The notation $\overline{\mathcal{G}^{\prime}(u)}$ is justified, since the operator $\overline{\mathcal{G}^{\prime}(u)}: Y \rightarrow \mathbb{R}^{m}$ is the closure of $A=\mathcal{G}^{\prime}(u)$ on the Banach space $Y$ with $D(A)=V$. 
Remark 1.6. (i) Note that we could apply Theorem 1.2 in the situation of Theorem 1.4 as well, but (3) yields a sharper estimate: If $Z=Y$ with $Y$ as in Theorem 1.4, then for $u, \bar{u} \in \mathcal{M}$ with $\|u-\bar{u}\|_{V} \leq \sigma$, we have

$$
\left\|\mathcal{E}^{\prime}(u)\right\|_{\overline{\mathcal{T}}_{u} \mathcal{M}^{*}}=\sup _{0 \neq y \in \overline{\mathcal{T}}_{u} \mathcal{M}} \frac{\mathcal{E}^{\prime}(u) y}{\|y\|_{Y}} \leq \sup _{0 \neq y \in \overline{\mathcal{T}_{u} \mathcal{M}}} \frac{\left\|\mathcal{E}^{\prime}(u)\right\|_{Y^{*}}\|y\|_{Y}}{\|y\|_{Y}}=\left\|\mathcal{E}^{\prime}(u)\right\|_{Y^{*}} .
$$

Thus, if the assumptions of Theorem 1.4 are satisfied and $C, \sigma, \theta$ are as in Theorem 1.4, we have $|\mathcal{E}(u)-\mathcal{E}(\bar{u})|^{1-\theta} \leq C\left\|\mathcal{E}^{\prime}(u)\right\|_{Y^{*}}$, i.e. (3) implies (2) under the assumptions of Theorem 1.4. It hence makes sense to refer to (3) as a refined Łojasiewicz-Simon gradient inequality.

(ii) From our proof, we cannot conclude that the Eojasiewicz exponents $\theta$ in Theorem 1.2 and Theorem 1.4 coincide.

Remark 1.7. The Hilbert space case treated in Corollary 5.2 is much easier to handle than Theorem 1.4. It is also more natural since one usually studies $H$-gradient flows with $H=W^{k, 2}(\Omega), \Omega \subset \mathbb{R}^{d}$ open, $k \in \mathbb{Z}$. On the other hand, one may sometimes encounter a problem in proving analyticity of the energy. The problematic phenomenon is, that whenever a Nemytskii or supercomposition operator

$$
\mathcal{F}: L^{p}(\Omega) \rightarrow L^{q}(\Omega), \quad \mathcal{F}(v)=f(v)=f \circ v \text { with } p, q \in[1, \infty)
$$

is analytic, the function $f$ has to be a polynomial of degree at most $\left\lceil\frac{p}{q}\right\rceil$, see [5, Theorem 3.16]. A way to work around this, is to choose suitable Sobolev spaces, such that all derivatives in the energy either appear in polynomial expressions with appropriate powers or are continuous. This is exactly why we work in the Banach space $W^{2, p}(\Omega)$ with $p>d$ to prove the Eojasiewicz-Simon gradient inequality in Section 7.1 .

This article is structured as follows. First, we recall some basic definitions and fundamental properties of analytic functions and Fredholm operators. Then we present the generalizations of basic concepts of differential geometry to submanifolds of a Banach space. In Section [3, we establish a local graph representation for the manifold $\mathcal{M}$ in Theorem 1.4. It turns out that studying this chart plays a crucial role in the proof of Theorem 1.4 which we complete in Section 4. After that, we consider the Hilbert space case in Section 5 in which the inequality takes a more convenient form. We also prove an abstract convergence result for the associated gradient flow in this case. Section 6 is dedicated to discuss the necessity of the assumptions we make in Theorem 1.4. In the last section, we will then apply our abstract results to the area of graph surfaces with an isoperimetric constraint in Section 7.1, the Allen-Cahn equation in Section 7.2 and to surfaces of revolution with prescribed volume in Section 7.3 . 


\section{Preliminaries}

\subsection{Analyticity}

Definition 2.1. Let $V, W$ be (real) Banach spaces, $D \subset V$ be an open set. A function $f: D \rightarrow W$ is called (real) analytic at $u_{0} \in D$ if there exist $\rho>0$ and continuous $\mathbb{R}$-multilinear forms $a_{n}: V^{n}:=\underbrace{V \times \cdots \times V}_{n \text {-times }} \rightarrow W$ for all $n \in \mathbb{N}_{0}$ such that

$$
\sum_{n=0}^{\infty}\left\|a_{n}\right\|_{\mathcal{L}\left(V^{n}, W\right)}\left\|u-u_{0}\right\|_{V}^{n} \text { converges and } f(u)=\sum_{n=0}^{\infty} a_{n}\left(u-u_{0}\right)^{n} \text { in } W
$$

for all $\left\|u-u_{0}\right\|_{V}<\rho$, where $a_{n}\left(u-u_{0}\right)^{n}:=a_{n}\left(u-u_{0}, \ldots, u-u_{0}\right) \in W$. The function $f$ is (real) analytic (on $D$ ) if it is analytic at every point $u_{0} \in D$.

We denote by $\mathcal{C}^{\omega}(D ; W)$ the vector space of analytic functions from $D$ to $W$. Like in the finite-dimensional case, a composition of two analytic maps is analytic.

Theorem 2.2 ([34, p. 1079]). Let $V, W, X$ be Banach spaces, $D \subset V$ and $E \subset W$ be open and $f: D \rightarrow W, g: E \rightarrow X$ be analytic with $f(D) \subset E$. Then $g \circ f: D \rightarrow X$ is analytic.

Easy examples of analytic maps are bounded multilinear maps.

Example 2.3. Let $\ell \in \mathbb{N}$ and $V_{1}, \ldots, V_{\ell}, W$ be Banach spaces. If $a: V_{1} \times \cdots \times V_{\ell} \rightarrow W$ is multilinear and continuous, then it is analytic. This follows easily since the series in (41) consists of exactly one nonzero term and hence converges.

\subsection{Fredholm operators}

Definition 2.4. Let $V, W$ be Banach spaces. An operator $T \in \mathcal{L}(V, W)$ is called a Fredholm operator if both $\operatorname{dim} \operatorname{ker} T$ and $\operatorname{codim}(\operatorname{Im} T, W)=\operatorname{dim}(W / \operatorname{Im} T)$ are finite. The number ind $T:=\operatorname{dim} \operatorname{ker} T-\operatorname{codim}(\operatorname{Im} T, W)$ is called the Fredholm index of $T$.

In the following, we collect some important properties of Fredholm operators.

Proposition 2.5 ([24, XVII, Corollaries 2.6 and 2.7]). Let $T \in \mathcal{L}(V, W)$ be a Fredholm operator. Then

(i) the image $\operatorname{Im} T \subset W$ is closed,

(ii) for any compact operator $K: V \rightarrow W$, the perturbed operator $T+K$ is Fredholm with ind $(T+K)=\operatorname{ind} T$. This holds in particular if $K$ has finite rank.

Theorem 2.6 ([24, XVII, Theorem 2.8]). Let $V, W$ and $X$ be Banach spaces and let $T \in \mathcal{L}(V, W)$ and $S \in \mathcal{L}(W, X)$ be Fredholm operators. Then $S \circ T \in \mathcal{L}(V, X)$ is a Fredholm operator and its index is given by ind $(S \circ T)=\operatorname{ind} S+\operatorname{ind} T$. 


\subsection{Complemented subspaces}

Projection operators and complemented subspaces play a crucial role in the proof of the Eojasiewicz-Simon gradient inequality in [10] and they will also be important for our result, specifically when investigating the properties of the submanifold $\mathcal{M}$ in Theorem 3.1

Definition and Lemma 2.7. A closed subspace $V_{0}$ of a Banach space $V$ is called complemented in $V$ if there exists a projection $P \in \mathcal{L}(V)$ with $\operatorname{Im} P=V_{0}$. Equivalently, there exists a closed subspace $V_{1}$ of $V$ with $V=V_{0} \oplus V_{1}$, see [8, Section 2.4].

Whereas in a Hilbert space, every closed subspace is complemented via the orthogonal projection (cf. [8, Chapter 5.1]), this is not true for a general Banach space. In fact, if in a Banach space $V$, every closed subspace is complemented, then it has to be isomorphic to a Hilbert space, see [26]. Nevertheless, some subspaces are always complemented.

Lemma 2.8 ([24, XV, Corollary 1.6]). Let $V$ be a Banach space and $V_{0} \subset V$ be a closed subspace, such that $\operatorname{dim} V_{0}<\infty$ or $\operatorname{codim}\left(V_{0}, V\right)<\infty$. Then $V_{0}$ is complemented in $V$.

\subsection{Submanifolds of Banach spaces}

This section is devoted to review some basic definitions in differential geometry in the setting of infinite-dimensional manifolds. Since we are only interested in the case of a submanifold of a Banach space $V$, the following definition based on [1, Definition 3.2.1] is sufficient for our purposes.

Definition 2.9. Let $V$ be a Banach space. A subset $\mathcal{M} \subset V$ is called a (splitting) submanifold of $V$ (of class $\mathcal{C}^{\ell}$ ) if for all $u \in \mathcal{M}$, there exists an open neighborhood $U \subset V$ of $u$, a complemented subspace $V_{0} \subset V$ and a map $\alpha \in \mathcal{C}^{\ell}(U ; V)$ which is a diffeomorphism onto its image, such that $\alpha(U \cap \mathcal{M})=\alpha(U) \cap V_{0}$. If $\alpha \in \mathcal{C}^{\omega}(U ; V)$, we say that $\mathcal{M}$ is analytic.

Example 2.10. If $V$ is a Banach space, $V_{0} \subset V$ is a complemented subspace, with $V=V_{0} \oplus V_{1}, \Omega_{0} \subset V_{0}$ is an open set and $\psi \in \mathcal{C}^{\ell}\left(\Omega_{0} ; V\right)$ with $\psi\left(\Omega_{0}\right) \subset V_{1}$, then $\mathcal{M}:=\left\{\omega+\psi(\omega) \mid \omega \in \Omega_{0}\right\}$ is a submanifold of $V$ of class $\mathcal{C}^{\ell}$.

Indeed, let $\Omega:=\Omega_{0}+V_{1}$ and write $\Omega \ni v=\omega+v_{1}$ with $\omega \in \Omega_{0}$ and $v_{1} \in V_{1}$ and define $\alpha: \Omega \rightarrow V, \alpha\left(\omega+v_{1}\right)=\omega+\left(v_{1}-\psi(\omega)\right) \in V_{0} \oplus V_{1}$. Then $\alpha$ is of class $\mathcal{C}^{\ell}$ and

$$
\alpha^{\prime}(\omega)\left[\begin{array}{l}
v_{0} \\
v_{1}
\end{array}\right]=\left[\begin{array}{cc}
\operatorname{Id}_{V_{0}} & 0 \\
-\psi^{\prime}(x) & \operatorname{Id}_{V_{1}}
\end{array}\right]\left[\begin{array}{l}
v_{0} \\
v_{1}
\end{array}\right] \text { for all } \omega \in \Omega_{0}, v_{0}+v_{1} \in V_{0} \oplus V_{1},
$$

so $\alpha^{\prime}(\omega): V \rightarrow V$ is an isomorphism. Since $\alpha$ is clearly bijective onto its image, we conclude that $\alpha$ is a $\mathcal{C}^{\ell}$-diffeomorphism by the Inverse Function Theorem 24, XIV, Theorem 1.2]. Consequently,

$$
\alpha(\Omega \cap M)=\alpha\left(\left\{\omega+\psi(\omega) \mid \omega \in \Omega_{0}\right\}\right)=\left\{\omega+\psi(\omega)-\psi(\omega) \mid \omega \in \Omega_{0}\right\}=\alpha(\Omega) \cap V_{0},
$$

thus $\mathcal{M}$ is a submanifold in the sense of Definition 2.9. 
Definition 2.11. Let $\mathcal{M} \subset V$ be a submanifold of class $\mathcal{C}^{\ell}$ with $\ell \geq 1$. The tangent space $\mathcal{T}_{u} \mathcal{M}$ of $\mathcal{M}$ at $u \in \mathcal{M}$ is defined by

$$
\mathcal{T}_{u} \mathcal{M}:=\left\{\gamma^{\prime}(0) \mid \exists \varepsilon>0, \gamma \in \mathcal{C}^{1}((-\varepsilon, \varepsilon) ; V) \text { with } \gamma(t) \in \mathcal{M} \forall t \in(-\varepsilon, \varepsilon) \text { and } \gamma(0)=u\right\} \text {. }
$$

Like in the finite-dimensional case, $\mathcal{T}_{u} \mathcal{M} \subset V$ is a subspace. We define the codimension of $\mathcal{M}$ in $V$ to be the codimension $\operatorname{codim}\left(\mathcal{T}_{u} \mathcal{M}, V\right)$ of $\mathcal{T}_{u} \mathcal{M}$ in $V$. The dual of the tangent space is called cotangent space and denoted by $\mathcal{T}_{u}^{*} \mathcal{M}:=\left(\mathcal{T}_{u} \mathcal{M}\right)^{*}$.

Definition and Lemma 2.12. Let $V$ be a Banach space, $U \subset V$ be an open set, $\emptyset \neq M \subset U$ and $\mathcal{E} \in \mathcal{C}^{1}(U ; \mathbb{R})$. We say that $\bar{u}$ is a constraint critical point of $\mathcal{E}$ on $M$ or $a$ critical point of $\left.\mathcal{E}\right|_{\mathcal{M}}$, if for any curve $\gamma \in \mathcal{C}^{1}((-\varepsilon, \varepsilon) ; V)$ with $\gamma(0)=\bar{u}$ and $\gamma(t) \in M$ for all $t \in(-\varepsilon, \varepsilon)$, the map $t \mapsto(\mathcal{E} \circ \gamma)(t)$ has a critical point at $t=0$.

If $M=\mathcal{M} \subset V$ is a submanifold, then $\bar{u} \in \mathcal{M}$ is a constraint critical point if and only if

$$
\mathcal{E}^{\prime}(\bar{u}) v=0 \text { for all } v \in \mathcal{T}_{\bar{u}} \mathcal{M} \subset V .
$$

Proof. This follows since for each curve $\gamma \in \mathcal{C}^{1}((-\varepsilon, \varepsilon) ; V)$ with $\gamma(0)=\bar{u}$ and $\gamma(t) \in \mathcal{M}$ for all $t \in(-\varepsilon, \varepsilon)$, we have $0=\left.\frac{\mathrm{d}}{\mathrm{d} t}\right|_{t=0}(\mathcal{E} \circ \gamma)(t)=\mathcal{E}^{\prime}(\bar{u}) \gamma^{\prime}(0)$.

\section{Local representation by a graph}

In this section, we will lay the foundations for the proof of our main theorem. We will see that the level set manifold $\mathcal{M}$ in Theorem 1.4 admits a natural chart around $\bar{u}$ representing $\mathcal{M}$ locally as a graph. After that, we will carefully analyze the properties of this induced chart.

For the rest of the article, we assume that $V$ and $Y$ are Banach spaces with $V \hookrightarrow Y$ densely, thus we get an induced embedding $Y^{*} \hookrightarrow V^{*}$. Furthermore, we assume that $U \subset V$ is an open set, $m \in \mathbb{N}$ and $\mathcal{G}: U \rightarrow \mathbb{R}^{m}$ is analytic. We study the nodal set of $\mathcal{G}$ given by $\mathcal{M}:=\{u \in U \mid \mathcal{G}(u)=0\}$.

Theorem 3.1. Let $\bar{u} \in \mathcal{M}$ such that $\mathcal{G}^{\prime}(\bar{u}): V \rightarrow \mathbb{R}^{m}$ is surjective. Then $V=V_{0} \oplus V_{1}$ with $V_{0}=\operatorname{ker} \mathcal{G}^{\prime}(\bar{u})$ for a closed subspace $V_{1} \subset V$. Moreover, there exist open sets $\Omega_{0} \subset V_{0}, \Omega_{1} \subset V_{1}$ with $\bar{u} \in \Omega=\Omega_{0} \times \Omega_{1} \subset U$ and an analytic function $\psi: \Omega_{0} \rightarrow V$ with $\psi\left(\Omega_{0}\right)=\Omega_{1}$ such that

$$
\mathcal{M} \cap \Omega=\left\{\omega+\psi(\omega) \mid \omega \in \Omega_{0}\right\}
$$

Hence, locally around $\bar{u}, \mathcal{M}$ is an analytic submanifold of $V$. Moreover, with $\varphi: \Omega_{0} \rightarrow V$, $\varphi(\omega):=\omega+\psi(\omega)$ we have for any $\omega \in \Omega_{0}, v \in V_{0}$

$$
\begin{aligned}
\psi^{\prime}(\omega) v & =-\left(\frac{\partial \mathcal{G}}{\partial v_{1}}(\varphi(\omega))\right)^{-1} \circ \mathcal{G}^{\prime}(\varphi(\omega)) v, \\
\varphi^{\prime}(\omega) v & =v-\left(\frac{\partial \mathcal{G}}{\partial v_{1}}(\varphi(\omega))\right)^{-1} \circ \mathcal{G}^{\prime}(\varphi(\omega)) v,
\end{aligned}
$$

where $\frac{\partial \mathcal{G}}{\partial v_{1}}(u):=\left.\mathcal{G}^{\prime}(u)\right|_{V_{1}}: V_{1} \rightarrow \mathbb{R}^{m}$ for $u \in U$. 
Proof. Since $\mathbb{R}^{m}=\operatorname{Im} \mathcal{G}^{\prime}(\bar{u}) \cong V / V_{0}, V_{0}$ has finite codimension in $V$. Moreover, since $V_{0}$ is closed by continuity, it is complemented by Lemma 2.8, i.e. there exists $V_{1} \subset V$ closed with $V=V_{0} \oplus V_{1}$. As a consequence thereof, $\frac{\partial \mathcal{G}}{\partial v_{1}}(\bar{u}): V_{1} \rightarrow \mathbb{R}^{m}$ is an isomorphism of Banach spaces. Thus, by the Implicit Function Theorem [35, Theorem 4.B], there exist open neighborhoods $\Omega_{0} \subset V_{0}, \Omega_{1} \subset V_{1}$ with $\Omega:=\Omega_{0} \times \Omega_{1} \subset U$ and $u \in \Omega$ such that for any $\omega \in \Omega_{0}$, there exists exactly one $\psi(\omega) \in \Omega_{1}$ with $\mathcal{G}(\omega+\psi(\omega))=0$. Analyticity of $\psi$ follows since $\mathcal{G}$ is analytic. By Example 2.10, we may conclude that $\mathcal{M} \cap \Omega$ is an analytic submanifold of $V$.

Moreover, the subset of invertible operators in $\mathcal{L}\left(V_{1}, \mathbb{R}^{m}\right)$ is open in the norm topology and the map $\left.\Omega \ni u \mapsto \mathcal{G}^{\prime}(u)\right|_{V_{1}} \in \mathcal{L}\left(V_{1}, \mathbb{R}^{m}\right)$ is continuous. Hence, by continuity, we can assume that $\frac{\partial \mathcal{G}}{\partial v_{1}}(u): V_{1} \rightarrow \mathbb{R}^{m}$ is an isomorphism for all $u \in \Omega$, passing to a smaller $\Omega$ if necessary. Therefore, (6) and thus (5) follow by differentiating the equation $0=\mathcal{G}(\omega+\psi(\omega))=\mathcal{G}(\varphi(\omega))$ for $\omega \in \Omega_{0}$.

Remark 3.2. (i) The relation $\mathcal{M} \cap \Omega=\left\{\omega+\psi(\omega) \mid \omega \in \Omega_{0}\right\}$ implies that the map $\varphi: \Omega_{0} \rightarrow \Omega \cap \mathcal{M}, \varphi(\omega)=\omega+\psi(\omega)$ defines a chart for $\mathcal{M} \cap \Omega$ centered at $\bar{u} \in \mathcal{M}$. Resembling the finite-dimensional case, we can identify $\omega+\psi(\omega)=(\omega, \psi(\omega))$ which means that $\mathcal{M}$ is locally the graph of $\psi$ near $\bar{u}$ (cf. Example 2.10).

(ii) Since we only work locally, we will abuse notation and speak about the manifold $\mathcal{M}$ instead of $\mathcal{M} \cap \Omega$ and write $\mathcal{T}_{u} \mathcal{M}$ for the tangent space $\mathcal{T}_{u}(\mathcal{M} \cap \Omega)$ at $u$.

The assumptions on $\mathcal{G}$ in Theorem 1.4 have some immediate consequences for the tangent space of $\mathcal{M}$.

Proposition 3.3. Suppose $\mathcal{G}: U \rightarrow \mathbb{R}^{m}$ and $\bar{u} \in \mathcal{M}$ satisfy assumptions (i), (iv) and (vi) in Theorem 1.4. Then, using the notation of Theorem 3.1, for $\omega \in \Omega_{0}, \varphi(\omega)=u$ we have

(i) $\mathcal{T}_{u} \mathcal{M}=\operatorname{ker} \mathcal{G}^{\prime}(u)=\operatorname{Im} \varphi^{\prime}(\omega)$,

(ii) $\overline{\mathcal{T}_{u} \mathcal{M}}:={\overline{\mathcal{T}_{u} \mathcal{M}}}^{\|\cdot\|_{Y}}=\operatorname{ker} \overline{\mathcal{G}^{\prime}(u)}$,

(iii) $\operatorname{codim}\left(\mathcal{T}_{u} \mathcal{M}, V\right)=\operatorname{codim}\left(\overline{\mathcal{T}_{u} \mathcal{M}}, Y\right)=m$ for all $u \in \Omega$.

Proof. (i) We first prove the inclusion $\mathcal{T}_{u} \mathcal{M} \subset \operatorname{ker} \mathcal{G}^{\prime}(u)$. Let $v \in \mathcal{T}_{u} \mathcal{M}$. Then, there exist $\varepsilon>0$ and $\gamma \in \mathcal{C}^{1}((-\varepsilon, \varepsilon), V)$ with $\operatorname{Im} \gamma \subset \mathcal{M} \cap \Omega, \gamma(0)=u$ and $\gamma^{\prime}(0)=v$. Now, $\mathcal{G}^{\prime}(u) v=\left.\frac{\mathrm{d}}{\mathrm{d} t}\right|_{t=0} \mathcal{G}(\gamma(t))=0$, since $\mathcal{M}=\{u \in U \mid \mathcal{G}(u)=0\}$.

For $\operatorname{ker} \mathcal{G}^{\prime}(u) \subset \operatorname{Im} \varphi^{\prime}(\omega)$, let $v \in V$ with $\mathcal{G}^{\prime}(u) v=0$ and write $v=v_{0}+v_{1} \in V_{0} \oplus V_{1}$. Then $0=\frac{\partial \mathcal{G}}{\partial v_{0}}(u) v_{0}+\frac{\partial \mathcal{G}}{\partial v_{1}}(u) v_{1}$. With $\varphi, \psi$ as in Theorem 3.1 and writing $u=\varphi(\omega)$ we conclude using (5)

$$
v_{1}=-\left(\frac{\partial \mathcal{G}}{\partial v_{1}}(u)\right)^{-1} \frac{\partial \mathcal{G}}{\partial v_{0}}(u) v_{0}=\psi^{\prime}(\omega) v_{0}
$$

Consequently, $\varphi^{\prime}(\omega) v_{0}=v_{0}+\psi^{\prime}(\omega) v_{0}=v_{0}+v_{1}=v$, thus $v \in \operatorname{Im} \varphi^{\prime}(\omega)$. 
To prove $\operatorname{Im} \varphi^{\prime}(\omega) \subset \mathcal{T}_{u} \mathcal{M}$, let $\omega \in \Omega_{0}$ with $\varphi(\omega)=u$ and let $y \in \operatorname{Im} \varphi^{\prime}(\omega)$. Then there exists $v \in V_{0}$ with $y=\varphi^{\prime}(\omega) v$, hence $y=\varphi^{\prime}(\omega) v=\left.\frac{\mathrm{d}}{\mathrm{d} t}\right|_{t=0} \varphi(\omega+t v) \in \mathcal{T}_{u} \mathcal{M}$ since $\gamma(t):=\varphi(\omega+t v)$ defines a curve in $\mathcal{M}$ with $\gamma(0)=u$ and $\gamma^{\prime}(0)=\varphi^{\prime}(\omega) v$.

(ii) First, let $y \in \overline{\mathcal{T}_{u} \mathcal{M}}$ and $v_{n} \in \mathcal{T}_{u} \mathcal{M}$ with $v_{n} \rightarrow y$ in $Y$. Using the extension property (iv) in Theorem 1.4, we get $\overline{\mathcal{G}^{\prime}(u)} y=\lim _{n \rightarrow \infty} \mathcal{G}^{\prime}(u) v_{n}=0$ by (i).

Conversely, let $y \in Y$ such that $\overline{\mathcal{G}^{\prime}(u)} y=0$. Since $\frac{\partial \mathcal{G}}{\partial v_{1}}(u): V_{1} \rightarrow \mathbb{R}^{m}$ is an isomorphism by the proof of Theorem 3.1, we conclude that $\mathcal{G}^{\prime}(u): V \rightarrow \mathbb{R}^{m}$ is surjective. As a consequence, $\mathbb{R}^{m} \cong V / \operatorname{ker} \mathcal{G}^{\prime}(u)$, so codim $\operatorname{ker} \mathcal{G}^{\prime}(u)=m$ is finite. By Lemma 2.8, there exists a closed subspace $W=W(u)$ of $V$ with $V=\operatorname{ker} \mathcal{G}^{\prime}(u) \oplus W$. By density of $V$ in $Y$, there exists a sequence $\left(v_{n}\right) \subset V$ with $v_{n} \rightarrow y$ in $Y$. Thus, we may write $v_{n}=v_{n}^{0}+w_{n}$ with $v_{n}^{0} \in \operatorname{ker} \mathcal{G}^{\prime}(u)$ and $w_{n} \in W$. As a consequence, $\mathcal{G}^{\prime}(u) v_{n}=\mathcal{G}^{\prime}(u) w_{n} \rightarrow \overline{\mathcal{G}^{\prime}(u)} y=0$ in $\mathbb{R}^{m}$. Since $\left.\mathcal{G}^{\prime}(u)\right|_{W}: W \rightarrow \mathbb{R}^{m}$ is an isomorphism, we get $w_{n} \rightarrow 0$ in $W \subset V$, hence in $Y$. Thus, $y=\lim _{n \rightarrow \infty} v_{n}=\lim _{n \rightarrow \infty} v_{n}^{0}$ with $v_{n}^{0} \in \operatorname{ker} \mathcal{G}^{\prime}(u)=\mathcal{T}_{u} \mathcal{M}$ by (i).

(iii) First, since $\frac{\partial \mathcal{G}}{\partial v_{1}}(u): V_{1} \rightarrow \mathbb{R}^{m}$ is an isomorphism by the proof of Theorem 3.1, the operator $\mathcal{G}^{\prime}(u): V \rightarrow \mathbb{R}^{m}$ is surjective. Thus $\mathbb{R}^{m} \cong V / \operatorname{ker} \mathcal{G}^{\prime}(u)=V / \mathcal{T}_{u} \mathcal{M}$ by (i), so $\operatorname{codim}\left(\mathcal{T}_{u} \mathcal{M}, V\right)=m$. Moreover, since $\mathcal{G}^{\prime}(u): V \rightarrow \mathbb{R}^{m}$ is surjective, so is the extension $\overline{\mathcal{G}^{\prime}(u)}: Y \rightarrow \mathbb{R}^{m}$ and hence $\mathbb{R}^{m} \cong Y / \operatorname{ker} \overline{\mathcal{G}^{\prime}(u)}=Y / \overline{\mathcal{T}_{u} \mathcal{M}}$ by (ii), so $\operatorname{codim}\left(\overline{\mathcal{T}_{u} \mathcal{M}}, Y\right)=m$.

Remark 3.4. In particular, Proposition 3.3 and Lemma 2.8 imply that there exists a projection $P(\bar{u}): Y \rightarrow Y$ onto $\overline{\mathcal{T}_{\bar{u}} \mathcal{M}}=\overline{\operatorname{ker} \mathcal{G}^{\prime}(u)}=: \overline{V_{0}}$.

As a next step, we investigate the properties of the chart $\varphi$ defined in Theorem 3.1 under the assumptions on $\mathcal{G}$ in Theorem 1.4 .

Proposition 3.5. Suppose $\mathcal{G}$ satisfies assumptions (iv) and (vi) in Theorem 1.4. Using the notation of Theorem 3.1, we have

(i) for all $\omega \in \Omega_{0}$, the operator $\psi^{\prime}(\omega): V_{0} \rightarrow V$ defined in (5) extends to an operator $\overline{\psi^{\prime}(\omega)} \in \mathcal{L}(Y)$ such that $\sup _{\omega \in \Omega_{0}}\left\|\overline{\psi^{\prime}(\omega)}\right\|_{\mathcal{L}(Y)}<\infty$ and $\operatorname{Im} \overline{\psi^{\prime}(\omega)} \subset V_{1}$ is finitedimensional, replacing $\Omega_{0}$ with a smaller neighborhood if necessary,

(ii) for any $\omega \in \Omega_{0}$, the operator $\varphi^{\prime}(\omega)$ extends to $\overline{\varphi^{\prime}(\omega)}=\operatorname{Id}_{Y}+\overline{\psi^{\prime}(\omega)}: Y \rightarrow Y$,

(iii) the map $\overline{\varphi^{\prime}}: \Omega_{0} \rightarrow \mathcal{L}(Y), \omega \mapsto \overline{\varphi^{\prime}(\omega)}$ is analytic,

(iv) $\overline{\varphi^{\prime}(\bar{\omega})} y=y$ for all $y \in \overline{V_{0}}$, where $\bar{\omega} \in \Omega_{0}$ satisfies $\varphi(\bar{\omega})=\bar{u}$.

(v) For all $\omega \in \Omega_{0}$ and $y \in \overline{V_{0}}$, we have $\left\|\overline{\varphi^{\prime}(\omega)} y\right\|_{Y} \geq \frac{1}{2}\|y\|_{Y}$, passing to a smaller neighborhood $\Omega_{0}$ if necessary.

Proof. (i) By assumption (iv) in Theorem 1.4, the operator $\mathcal{G}^{\prime}(\varphi(\omega))$ extends to an operator $\overline{\mathcal{G}^{\prime}(\varphi(\omega))}: Y \rightarrow \mathbb{R}^{m}$ and hence $\psi^{\prime}(\omega)$ extends to an operator $\overline{\psi^{\prime}(\omega)}: Y \rightarrow Y$ via (5). Since the image of $\left(\frac{\partial \mathcal{G}}{\partial v_{1}}(\varphi(\omega))\right)^{-1}: \mathbb{R}^{m} \rightarrow V_{1}$ is contained in $V_{1}$, we conclude 
that $\operatorname{Im} \overline{\psi^{\prime}(\omega)} \subset V_{1} \subset Y$ is finite-dimensional. For the norm estimate note that for any $y \in Y$, using $V \hookrightarrow Y$, we have

$$
\left\|\overline{\psi^{\prime}(\omega)} y\right\|_{Y} \leq C\left\|\left(\frac{\partial \mathcal{G}}{\partial v_{1}}(\varphi(\omega))\right)^{-1}\right\|_{\mathcal{L}\left(\mathbb{R}^{m}, V_{1}\right)}\left\|\overline{\mathcal{G}^{\prime}(\varphi(\omega))}\right\|_{\mathcal{L}\left(Y, \mathbb{R}^{m}\right)}\|y\|_{Y} \leq C C^{\prime}\|y\|_{Y}
$$

for some $C, C^{\prime}>0$, passing to a smaller $\Omega_{0}$ if necessary, since $\varphi$ and $\mathcal{G}$ are analytic and so is the extension $\overline{\mathcal{G}^{\prime}}: U \rightarrow \mathcal{L}\left(Y, \mathbb{R}^{m}\right)$ by assumption (iv) in Theorem 1.4,

(ii) This follows from (i) and (6).

(iii) The map $\Omega_{0} \ni \omega \mapsto \overline{\mathcal{G}^{\prime}(\varphi(\omega))} \in \mathcal{L}\left(Y, \mathbb{R}^{m}\right)$ is analytic using assumption (iv) in Theorem 1.4 and the analyticity of $\varphi$. Therefore, using (6) , so is the extension $\Omega_{0} \ni \omega \mapsto \overline{\overline{\varphi^{\prime}(\omega)}} \in \mathcal{L}(Y)$ using Theorem 2.2 and Example 2.3.

(iv) By Proposition 3.3 (ii) and (5), $\overline{\psi^{\prime}(\omega)} y=0$ for all $y \in \overline{V_{0}}$. This yields the claim.

(v) By (iv), $\left\|\overline{\varphi^{\prime}(\bar{\omega})} y\right\|_{Y}=y$ for all $y \in \overline{V_{0}}$. By (iii), passing to a smaller $\Omega_{0}$ if necessary, we can assume $\left\|\overline{\varphi^{\prime}(\bar{\omega})}-\overline{\varphi^{\prime}(\omega)}\right\|_{\mathcal{L}(Y)} \leq \frac{1}{2}$ for all $w \in \Omega_{0}$. Then, for any $y \in \overline{V_{0}}$ we can estimate $\left\|\overline{\varphi^{\prime}(\omega)} y\right\|_{Y} \geq\left\|\overline{\varphi^{\prime}(\bar{\omega})} y\right\|_{Y}-\left\|\overline{\varphi^{\prime}(\bar{\omega})} y-\overline{\varphi^{\prime}(\omega)} y\right\|_{Y} \geq\left(1-\frac{1}{2}\right)\|y\|_{Y}$.

\section{Proof of the Łojasiewicz-Simon gradient inequality}

In this section, we will establish the Łojasiewicz-Simon gradient inequality for the energy $\mathcal{E}$ composed with the chart we constructed in Section 3 and use this to prove our main theorem.

Theorem 4.1. Suppose $\mathcal{E}, \mathcal{G}$ and $\bar{u} \in U$ satisfy assumptions (i), (ii), (iv) and (vi) of Theorem 1.4. Let $\varphi$ be the chart centered at $\bar{u}$ defined in Theorem [3.1. Define $\mathcal{F}: \Omega_{0} \rightarrow \mathbb{R}, \mathcal{F}(\omega):=(\mathcal{E} \circ \varphi)(\omega)$. Then

(i) $\mathcal{F}$ is analytic,

(ii) for $\omega \in \Omega_{0}, \mathcal{F}^{\prime}(\omega) \in{\overline{V_{0}}}^{*}$ via $\mathcal{F}^{\prime}(\omega)=P(\bar{u})^{*} \circ{\overline{\varphi^{\prime}(\omega)}}^{*} \mathcal{E}^{\prime}(\varphi(\omega))$, where $P(\bar{u}) \in \mathcal{L}(Y)$ is the projection onto $\overline{V_{0}}=\overline{\mathcal{T}_{\bar{u}} \mathcal{M}}$ from Remark 3.4.

(iii) the map $\Omega_{0} \ni \omega \mapsto \mathcal{F}^{\prime}(\omega) \in{\overline{V_{0}}}^{*}$ is analytic.

Proof. (i) This follows from Theorem 2.2 since $\mathcal{E}$ and $\varphi$ are analytic.

(ii) Let $\omega \in \Omega_{0}, v \in V_{0}$ and $P(\bar{u}) \in \mathcal{L}(Y)$ be as in Remark 3.4. We compute

$$
\begin{aligned}
\mathcal{F}^{\prime}(\omega) v & =\mathcal{E}^{\prime}(\varphi(\omega)) \circ \varphi^{\prime}(\omega) v=\mathcal{E}^{\prime}(\varphi(\omega)) \circ \overline{\varphi^{\prime}(\omega)} v \\
& ={\overline{\varphi^{\prime}(\omega)}}^{*} \circ \mathcal{E}^{\prime}(\varphi(\omega))(P(\bar{u}) v)=P(\bar{u})^{*} \circ{\overline{\varphi^{\prime}(\omega)}}^{*} \circ \mathcal{E}^{\prime}(\varphi(\omega)) v
\end{aligned}
$$

using that $P(\bar{u}) v=v$ since $v \in V_{0} \subset \overline{V_{0}}$. Since $P(\bar{u})$ projects onto ${\overline{V_{0}}}^{*}$, (ii) follows. 
(iii) The chart $\varphi: \Omega_{0} \rightarrow U$ is analytic and so is $\mathcal{E}^{\prime}: U \rightarrow Y^{*}$ by assumption (ii) in Theorem 1.4. Thus, so is their composition $\omega \mapsto \mathcal{E}^{\prime}(\varphi(\omega)): \Omega_{0} \rightarrow Y^{*}$ by Theorem 2.2. By Proposition [3.5, the extension $\overline{\varphi^{\prime}}: \Omega_{0} \rightarrow \mathcal{L}(Y)$ is analytic, and so is taking the adjoint $T \mapsto T^{*}: \mathcal{L}(Y) \rightarrow \mathcal{L}\left(Y^{*}\right)$ by Example 2.3, since it is linear and bounded. Similarly, the evaluation map $\mathcal{L}\left(Y^{*}\right) \times Y^{*} \rightarrow Y^{*},\left(T, y^{*}\right) \mapsto T y^{*}$ is analytic. Therefore, $\Omega_{0} \rightarrow{\overline{V_{0}}}^{*}, \omega \mapsto \mathcal{F}^{\prime}(\omega)=P(\bar{u}) \circ{\overline{\varphi^{\prime}(\omega)}}^{*} \circ \mathcal{E}^{\prime}(\varphi(\omega))$ is analytic, since the projection $P(\bar{u}): Y \rightarrow{\overline{V_{0}}}^{*}$ is analytic.

The following lemma justifies our approach to study $\mathcal{F}$ in order to prove Theorem 1.4 .

Lemma 4.2. Suppose $\mathcal{E}$ and $\mathcal{G}$ are analytic and satisfy assumptions (i), (ii), (iv) and (vi) in Theorem 1.4. Let $\bar{u} \in U$ and let $\varphi$ be the chart centered at $\bar{u}$ defined in Theorem 3.1. Let $\bar{\omega} \in \Omega_{0}$ such that $\varphi(\bar{\omega})=\bar{u}$ and $\mathcal{F}=\mathcal{E} \circ \varphi$ as in Theorem 4.1. Then the following are equivalent.

(i) $\mathcal{F}$ satisfies a Eojasiewicz-Simon gradient inequality at $\bar{\omega}$, i.e. there exist $C, \sigma^{\prime}>0$ and $\theta \in\left(0, \frac{1}{2}\right]$ such that

$$
|\mathcal{F}(\omega)-\mathcal{F}(\bar{\omega})|^{1-\theta} \leq C\left\|\mathcal{F}^{\prime}(\omega)\right\|_{\bar{V}_{0}} \text { for all }\|\omega-\bar{\omega}\|_{V} \leq \sigma^{\prime} .
$$

(ii) $\left.\mathcal{E}\right|_{\mathcal{M}}$ satisfies a refined Lojasiewicz-Simon gradient inequality (3) near $\bar{u}$.

Proof. Suppose (i) holds. Let $u \in \mathcal{M}$ with $\|u-\bar{u}\|_{V} \leq \sigma$. For $\sigma>0$ small enough, we can assume $u \in \Omega, u=\varphi(\omega)$ for a unique $\omega \in \Omega_{0}$ and $\|\omega-\bar{\omega}\|_{V} \leq \sigma^{\prime}$ by continuity. Then by (i), we have

$$
|\mathcal{E}(u)-\mathcal{E}(\bar{u})|^{1-\theta}=|\mathcal{F}(\omega)-\mathcal{F}(\bar{\omega})|^{1-\theta} \leq C\left\|\mathcal{F}^{\prime}(\omega)\right\|_{{\overline{V_{0}}}^{*}} .
$$

Now, by Proposition [3.3, we have $\mathcal{T}_{\varphi(\omega)} \mathcal{M}=\operatorname{Im} \varphi^{\prime}(\omega)$ and thus $\varphi^{\prime}(\omega) v \in \mathcal{T}_{\varphi(\omega)} \mathcal{M}$ for $v \in V_{0}$. By continuity of the extension (see Proposition $\overline{3.5}$ (ii)), we get $\overline{\varphi^{\prime}(\omega)} y \in \overline{\mathcal{T}_{\varphi(\omega)} \mathcal{M}}$ for $y \in \overline{V_{0}}$. Using $P(\bar{u}) y=y$ for $y \in \overline{V_{0}}$ and Theorem 4.1 (ii), we compute

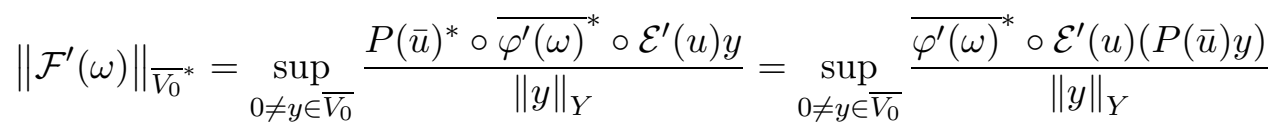

$$
\begin{aligned}
& =\sup _{0 \neq y \in \overline{V_{0}}} \frac{\mathcal{E}^{\prime}(u)\left(\overline{\varphi^{\prime}(\omega)} y\right)}{\|y\|_{Y}} \leq \sup _{\substack{0 \neq y \in \overline{V_{0}}\\
}} \frac{\left\|\mathcal{E}^{\prime}(u)\right\|_{\overline{\mathcal{T}_{u} \mathcal{M}}} *\left\|\overline{\varphi^{\prime}(\omega)} y\right\|_{\overline{\mathcal{T}_{u} \mathcal{M}}}}{\|y\|_{Y}} \\
& \leq\left\|\mathcal{E}^{\prime}(u)\right\|_{\overline{\mathcal{T}_{u} \mathcal{M}^{*}}} \sup _{0 \neq y \in \overline{V_{0}}} \frac{\left\|\overline{\varphi^{\prime}(\omega)} y\right\|_{Y}}{\|y\|_{Y}} \leq\left\|\mathcal{E}^{\prime}(u)\right\|_{\overline{\mathcal{T}}_{u} \mathcal{M}^{*}} \sup _{\omega \in \Omega_{0}}\left\|\overline{\varphi^{\prime}(\omega)}\right\|_{\mathcal{L}(Y)} .
\end{aligned}
$$

Now, reducing $\sigma, \sigma^{\prime}>0$ if necessary, and using Proposition 3.5 (i), we may assume that $\sup _{v \in \Omega_{0}}\left\|\overline{\varphi^{\prime}(v)}\right\|_{\mathcal{L}(Y)} \leq 1+\sup _{v \in \Omega_{0}}\left\|\overline{\psi^{\prime}(v)}\right\|_{\mathcal{L}(Y)} \leq C^{\prime}<\infty$. Hence, using (17) and (8), we conclude $|\mathcal{E}(u)-\mathcal{E}(\bar{u})|^{1-\theta} \leq C C^{\prime}\left\|\mathcal{E}^{\prime}(u)\right\|_{\overline{\mathcal{T}}_{u} \mathcal{M}^{*}}$. 
Conversely, suppose $\left.\mathcal{E}\right|_{\mathcal{M}}$ satisfies (3) for some $C, \sigma>0$ and $\theta \in\left(0, \frac{1}{2}\right]$. Let $\omega \in \Omega_{0}$ with $\|\omega-\bar{\omega}\|_{V} \leq \sigma^{\prime}$. Define $u:=\varphi(\omega)$ and let $\sigma^{\prime}>0$ be small enough such that $\|u-\bar{u}\|_{V} \leq \sigma$. Then we have

$$
|\mathcal{F}(\omega)-\mathcal{F}(\bar{\omega})|^{1-\theta}=|\mathcal{E}(u)-\mathcal{E}(\bar{u})|^{1-\theta} \leq C\left\|\mathcal{E}^{\prime}(u)\right\|_{\overline{\mathcal{T}}_{\mathcal{M}^{\mathcal{M}}}}{ } .
$$

Now, fix $0 \neq w \in \mathcal{T}_{u} \mathcal{M}$. Then by Proposition 3.3, $w=\varphi^{\prime}(\omega) v$ for some $0 \neq v \in V_{0}$. We have

$$
\mathcal{E}^{\prime}(u) w=\mathcal{E}^{\prime}(u)\left(\varphi^{\prime}(\omega) v\right)=\mathcal{E}^{\prime}(u)\left({\overline{\varphi^{\prime}(\omega)}}\right)={\overline{\varphi^{\prime}(\omega)}}^{*} \circ \mathcal{E}^{\prime}(u)(P(\bar{u}) v)=\mathcal{F}^{\prime}(\omega) v
$$

using $P(\bar{u}) v=v$ since $v \in \overline{V_{0}}$ and Theorem 4.1 (ii). Thus, we find

$$
\frac{\mathcal{E}^{\prime}(u) w}{\|w\|_{Y}}=\frac{\mathcal{F}^{\prime}(\omega) v}{\left\|\overline{\varphi^{\prime}(\omega)} v\right\|_{Y}} \leq 2 \frac{\mathcal{F}^{\prime}(\omega) v}{\|v\|_{Y}} \leq 2 \sup _{\substack{0 \neq v \in V_{0}\\}} \frac{\mathcal{F}^{\prime}(\omega) v}{\|v\|_{Y}} \leq 2 \sup _{\substack{0 \neq y \in \overline{V_{0}}\\}} \frac{\mathcal{F}^{\prime}(\omega) y}{\|y\|_{Y}}=2\left\|\mathcal{F}^{\prime}(\omega)\right\|_{\bar{V}_{0}}{ },
$$

using Proposition 3.5 (v) and reducing $\sigma^{\prime}>0$ if necessary. Since $\mathcal{E}^{\prime}(u) \in Y^{*}$ by assumption, we may conclude that (10) remains valid if $0 \neq w \in \overline{\mathcal{T}_{u} \mathcal{M}}$ by continuity. Combining this with (9), the claim follows.

Theorem 4.3. Suppose $\mathcal{E}, \mathcal{G}$ and $\bar{u} \in U$ satisfy the assumptions of Theorem 1.4 above and let $\mathcal{F}$ be as in Theorem 4.1. Then, for $\bar{\omega} \in \Omega_{0}$ with $\varphi(\bar{\omega})=\bar{u}$, the operator $\mathcal{F}^{\prime \prime}(\bar{\omega}): V_{0} \rightarrow{\overline{V_{0}}}^{*}$ is Fredholm of index zero.

Proof. Let $\varphi(\bar{\omega})=\bar{u}$ and $v \in V_{0}$. By Proposition 3.5 (iv), we have $\overline{\varphi^{\prime}(\omega)} v=v$. We use the chain rule, the analyticity of $\varphi, \psi, \overline{\varphi^{\prime}}, \overline{\psi^{\prime}}$ and $\mathcal{E}^{\prime}: U \rightarrow Y^{*}$, and the analyticity and bilinearity of the evaluation map $\mathcal{L}\left(Y^{*}\right) \times Y^{*} \rightarrow Y^{*}$ to compute

$$
\begin{aligned}
& \mathcal{F}^{\prime \prime}(\bar{\omega}) v=\left.\frac{\mathrm{d}}{\mathrm{d} t}\right|_{t=0} \mathcal{F}^{\prime}(\bar{\omega}+t v)=\left.P(\bar{u})^{*} \circ \frac{\mathrm{d}}{\mathrm{d} t}\right|_{t=0}\left({\overline{\varphi^{\prime}(\bar{\omega}+t v)}}^{*} \mathcal{E}^{\prime}(\varphi(\bar{\omega}+t v))\right) \\
& =\left.P(\bar{u})^{*} \circ \frac{\mathrm{d}}{\mathrm{d} t}\right|_{t=0}\left(\mathcal{E}^{\prime}(\varphi(\bar{\omega}+t v))+{\overline{\psi^{\prime}(\bar{\omega}+t v)}}^{*} \mathcal{E}^{\prime}(\varphi(\bar{\omega}+t v))\right) \\
& =P(\bar{u})^{*} \circ \mathcal{E}^{\prime \prime}(\bar{u}) v+P(\bar{u})^{*}\left(\left(\left.\frac{\mathrm{d}}{\mathrm{d} t}\right|_{t=0} \overline{\psi^{\prime}(\bar{\omega}+t v)}\right)^{*} \mathcal{E}^{\prime}(\bar{u})+{\overline{\psi^{\prime}(\bar{\omega})}}^{*} \mathcal{E}^{\prime \prime}(\bar{u}) v\right) \\
& =: P(\bar{u})^{*} \circ \mathcal{E}^{\prime \prime}(\bar{u}) v+E v \text {, }
\end{aligned}
$$

using Theorem 4.1 (ii), Proposition 3.5 and the fact that ${\overline{\varphi^{\prime}}}^{*}=\operatorname{Id}_{Y^{*}}+{\overline{\psi^{\prime}}}^{*}$ by (6). We will now show that $E: V_{0} \rightarrow{\overline{V_{0}}}^{*}$ is compact. First, using (5), we compute

$$
\begin{aligned}
\left.\frac{\mathrm{d}}{\mathrm{d} t}\right|_{t=0} \overline{\psi^{\prime}(\bar{\omega}+t v)} & =\left.\frac{\mathrm{d}}{\mathrm{d} t}\right|_{t=0}\left(-\left(\frac{\partial \mathcal{G}}{\partial v_{1}}(\varphi(\bar{\omega}+t v))\right)^{-1} \circ\left(\overline{\mathcal{G}^{\prime}(\varphi(\bar{\omega}+t v))}\right)\right) \\
& =-\left(\left.\frac{\mathrm{d}}{\mathrm{d} t}\right|_{t=0}\left(\frac{\partial \mathcal{G}}{\partial v_{1}}(\varphi(\bar{\omega}+t v))\right)^{-1}\right) \circ \overline{\mathcal{G}^{\prime}(\bar{u})}
\end{aligned}
$$




$$
\begin{aligned}
& -\left.\left(\frac{\partial \mathcal{G}}{\partial v_{1}}(\bar{u})\right)^{-1} \circ \frac{\mathrm{d}}{\mathrm{d} t}\right|_{t=0} \overline{\mathcal{G}^{\prime}(\varphi(\bar{\omega}+t v))} \\
= & -\left(\left.\frac{\mathrm{d}}{\mathrm{d} t}\right|_{t=0}\left(\frac{\partial \mathcal{G}}{\partial v_{1}}(\varphi(\bar{\omega}+t v))\right)^{-1}\right) \circ \overline{\mathcal{G}^{\prime}(\bar{u})} \\
& -\left(\frac{\partial \mathcal{G}}{\partial v_{1}}(\bar{u})\right)^{-1} \circ\left(\overline{\mathcal{G}^{\prime}}\right)^{\prime}(\bar{u}) v=:-R v-S v,
\end{aligned}
$$

where $R, S \in \mathcal{L}\left(V_{0}, \mathcal{L}(Y)\right)$. We conclude that

$$
\left(\left.\frac{\mathrm{d}}{\mathrm{d} t}\right|_{t=0} \overline{\psi^{\prime}(\bar{\omega}+t v)}\right)^{*} \mathcal{E}^{\prime}(\bar{u})=-(R v)^{*} \mathcal{E}^{\prime}(\bar{u})-(S v)^{*} \mathcal{E}^{\prime}(\bar{u}) .
$$

Recall that for $u \in \Omega=\varphi\left(\Omega_{0}\right),\left(\frac{\partial \mathcal{G}}{\partial v_{1}}(u)\right)^{-1}: \mathbb{R}^{m} \rightarrow V_{1} \hookrightarrow Y$. The first part of (12) is

$$
(R v)^{*} \mathcal{E}^{\prime}(\bar{u})=\left(\overline{\mathcal{G}^{\prime}(\bar{u})}\right)^{*} \circ\left(\left.\frac{\mathrm{d}}{\mathrm{d} t}\right|_{t=0}\left(\frac{\partial \mathcal{G}}{\partial v_{1}}(\varphi(\bar{\omega}+t v))\right)^{-1}\right)^{*} \mathcal{E}^{\prime}(\bar{u}),
$$

so the image of $v \mapsto(R v)^{*} \mathcal{E}^{\prime}(\bar{u})$ is contained in $\operatorname{Im}\left(\overline{\mathcal{G}^{\prime}(\bar{u}}\right)^{*} \subset Y^{*}$, which is finitedimensional since $\overline{\mathcal{G}^{\prime}(\bar{u})}: Y \rightarrow \mathbb{R}^{m}$ has finite rank.

Furthermore, with $\eta:=\left(\left(\frac{\partial \mathcal{G}}{\partial v_{1}}(\bar{u})\right)^{-1}\right)^{*} \mathcal{E}^{\prime}(\bar{u}) \in \mathbb{R}^{m}$ we have

$$
(S v)^{*} \mathcal{E}^{\prime}(\bar{u})=\left(\left(\overline{\mathcal{G}^{\prime}}\right)^{\prime}(\bar{u}) v\right)^{*} \circ\left(\left(\frac{\partial \mathcal{G}}{\partial v_{1}}(\bar{u})\right)^{-1}\right)^{*} \mathcal{E}^{\prime}(\bar{u})=\left(\left(\overline{\mathcal{G}^{\prime}}\right)^{\prime}(\bar{u}) v\right)^{*} \eta .
$$

We will now show that the operator $v \mapsto(S v)^{*} \mathcal{E}^{\prime}(\bar{u}): V_{0} \rightarrow Y^{*}$ is compact. Let $v_{n} \in V_{0}$ for $n \in \mathbb{N}$ with $\left\|v_{n}\right\|_{V} \leq 1$. By assumption (v) in Theorem 1.4. passing to a subsequence, we can assume $\left(\overline{\mathcal{G}^{\prime}}\right)^{\prime}(\bar{u}) v_{n} \rightarrow A$ in $\mathcal{L}\left(Y, \mathbb{R}^{m}\right)$. Since taking the adjoint is continuous, this yields $\left(\left(\overline{\mathcal{G}^{\prime}}\right)^{\prime}(\bar{u}) v_{n}\right)^{*} \rightarrow A^{*}$ in $\mathcal{L}\left(\mathbb{R}^{m}, Y^{*}\right)$. But this clearly implies $\left.\left(\overline{\mathcal{G}^{\prime}}\right)^{\prime}(\bar{u}) v_{n}\right)^{*} \eta \rightarrow A^{*} \eta$ in $Y^{*}$.

By the previous arguments, together with (12), (13) and (14), we conclude that the linear operator $V_{0} \rightarrow Y^{*}, v \mapsto\left(\left.\frac{\mathrm{d}}{\mathrm{d} t}\right|_{t=0} \overline{\psi^{\prime}(\bar{\omega}+t v)}\right)^{*} \mathcal{E}^{\prime}(\bar{u})$ is compact.

Clearly, since $\overline{\psi^{\prime}(\bar{\omega})}$ has finite rank, the image of $v \mapsto{\overline{\psi^{\prime}(\bar{\omega})}}^{*} \mathcal{E}^{\prime \prime}(\bar{u}) v$ is contained in $\operatorname{Im}\left(\overline{\psi^{\prime}(\bar{\omega})}\right)^{*}$ and thus finite-dimensional. As a consequence,

$$
E: V_{0} \rightarrow{\overline{V_{0}}}^{*}, E v=P(\bar{u})^{*}\left(-(R v)^{*} \mathcal{E}^{\prime}(\bar{u})-(S v)^{*} \mathcal{E}^{\prime}(\bar{u})+{\overline{\psi^{\prime}(\bar{\omega})}}^{*} \mathcal{E}^{\prime \prime}(\bar{u}) v\right)
$$

is a compact operator. We will now show that $\mathcal{F}^{\prime \prime}(\bar{\omega}): V_{0} \rightarrow{\overline{V_{0}}}^{*}$ is Fredholm with ind $\mathcal{F}^{\prime \prime}(\bar{\omega})=0$. By (11) and Proposition 2.5, it is enough to show that $T: V \mapsto{\overline{V_{0}}}^{*}$, $v \mapsto P(\bar{u})^{*} \circ \mathcal{E}^{\prime \prime}(\bar{u}) v$ is Fredholm of index zero.

Note that $T=P(\bar{u})^{*} \circ \mathcal{E}^{\prime \prime}(\bar{u}) \circ \iota$. Here, $\iota: V_{0} \hookrightarrow V$ is the inclusion, which is Fredholm since $\operatorname{ker} \iota=\{0\}$ and $\operatorname{codim}(\operatorname{Im} \iota, V)=\operatorname{codim}\left(V_{0}, V\right)=\operatorname{codim}\left(\mathcal{T}_{\bar{u}} \mathcal{M}, V\right)=m$ by 
Proposition 3.3. Moreover, recall from Remark 3.4 that $P(\bar{u}): Y \rightarrow Y$ is the projection onto $\overline{V_{0}}=\overline{\mathcal{T}_{\bar{u}} \mathcal{M}}$ with $\operatorname{codim}\left(\overline{V_{0}}, Y\right)=m$ by Proposition 3.3 . Thus $Y=\overline{V_{0}} \oplus Z$ with $\operatorname{dim} Z=m$. Then $\operatorname{ker} P(\bar{u})^{*} \cong Z^{*}$, so $\operatorname{dim} \operatorname{ker} P(\bar{u})^{*}=\operatorname{dim} Z^{*}=m$. Clearly, $\operatorname{codim}\left(\operatorname{Im} P(\bar{u})^{*},{\overline{V_{0}}}^{*}\right)=0$.

Therefore, by Theorem [2.6, the composition $T=P(\bar{u})^{*} \circ \mathcal{E}^{\prime \prime}(\bar{u}) \circ \iota$ is Fredholm with ind $T=$ ind $P(\bar{u})^{*}+$ ind $\mathcal{E}^{\prime \prime}(\bar{u})+$ ind $\iota=m+$ ind $\mathcal{E}^{\prime \prime}(\bar{u})-m=0$.

Now, it is not difficult to see that $\mathcal{F}$ satisfies a Łojasiewicz-Simon gradient inequality at a critical point by Theorem 1.2 .

Theorem 4.4. Suppose $\mathcal{E}, \mathcal{G}$ and $\bar{u} \in \mathcal{M}$ satisfy the assumptions of Theorem 1.4. Let $\varphi$ be the chart constructed in Theorem 3.1 with $\bar{v} \in \Omega_{0}$ such that $\varphi(\bar{\omega})=\bar{u}$. If $\overline{\mathcal{F}}^{\prime}(\bar{\omega})=0$, then there exist $C, \sigma^{\prime}>0$ and $\theta \in\left(0, \frac{1}{2}\right]$ such that

$$
|\mathcal{F}(\omega)-\mathcal{F}(\bar{\omega})|^{1-\theta} \leq C\left\|\mathcal{F}^{\prime}(\omega)\right\|_{\bar{V}_{0}} \text { for all }\|\omega-\bar{\omega}\|_{V} \leq \sigma^{\prime} .
$$

Proof. We verify that the assumptions in Theorem 1.2 are satisfied for $V=V_{0}, U=\Omega_{0}$ $Z=\overline{V_{0}}, \varphi=\bar{u}$ and $\mathcal{E}=\mathcal{F}$. Density of $V_{0} \subset \overline{V_{0}}$ is trivial.

Assumption (ii) in Theorem 1.2 is satisfied by Theorem 4.1 (iii). Assumption (iii), i.e. the Fredholm property of $\mathcal{F}^{\prime \prime}(\bar{\omega}): V_{0} \rightarrow{\overline{V_{0}}}^{*}$, holds by Theorem 4.3 . Hence, $\mathcal{F}$ satisfies a Łojasiewicz-Simon gradient inequality in a neighborhood of $\bar{\omega}$ by Theorem 1.2 ,

We are finally able to prove our main result.

Proof of Theorem 1.4. Suppose $\mathcal{E}, \mathcal{G}$ and $\bar{u} \in \mathcal{M}=\{u \in \mathcal{M} \mid \mathcal{G}(u)=0\}$ satisfy the assumptions of Theorem 1.4. Suppose $\bar{u}$ is a constraint critical point in the sense of Definition and Lemma 2.12, By Theorem 3.1, Remark 3.2 and Proposition 3.3, $\mathcal{M}$ is locally a manifold near $\bar{u}$ with codimension $m$. Let $\varphi: \Omega_{0} \rightarrow \Omega \cap \mathcal{M}$ be the chart from Theorem 3.1 centered at $\bar{u}$ with $\varphi(\bar{\omega})=\bar{u}$. Recall from Definition and Lemma 2.12 that $\mathcal{E}^{\prime}(\bar{u}) v=0$ for all $v \in \mathcal{T}_{\bar{u}} \mathcal{M}=\operatorname{Im} \varphi^{\prime}(\bar{\omega})$ by Proposition 3.3 (i). Then, for any $v \in V_{0}$, using Theorem 4.1 and $P(\bar{u}) v=v$ we have

$$
\begin{aligned}
\mathcal{F}^{\prime}(\bar{\omega}) v & =P(\bar{u})^{*} \circ{\overline{\varphi^{\prime}(\bar{\omega})}}^{*} \circ \mathcal{E}^{\prime}(\bar{u}) v={\overline{\varphi^{\prime}(\bar{\omega})}}^{*} \circ \mathcal{E}^{\prime}(\bar{u}) P(\bar{u}) v \\
& ={\overline{\varphi^{\prime}(\bar{\omega})}}^{*} \circ \mathcal{E}^{\prime}(\bar{u}) v=\mathcal{E}^{\prime}(\bar{u}) \varphi^{\prime}(\bar{\omega}) v=0 .
\end{aligned}
$$

Hence, by Theorem 4.4 there exist $C, \sigma^{\prime}>0$ and $\theta \in\left(0, \frac{1}{2}\right]$ such that $\mathcal{F}$ satisfies a Łojasiewicz-Simon gradient inequality at $\bar{\omega}$. By Lemma 4.2 the claim follows.

\section{The Hilbert space framework}

In the setting where $Y=Y^{*}=H$ is a Hilbert space, the assumptions in Theorem 1.4 can be characterized in a simpler way in terms of the $H$-gradients.

Definition and Lemma 5.1. Let $V$ be a Banach space and let $(H,\langle\cdot, \cdot\rangle)$ be a Hilbert space such that $V \hookrightarrow H$ densely, so $H \hookrightarrow V^{*}$. Suppose $U \subset V$ is an open set and $\mathcal{E} \in \mathcal{C}^{1}(U ; \mathbb{R})$. If $\mathcal{E}^{\prime}(u) \in H$ under the identification of $H$ with its image in $V^{*}$, we say 
that $\mathcal{E}$ possesses an $H$-gradient at $u \in U$ and we write $\nabla \mathcal{E}(u):=\mathcal{E}^{\prime}(u) \in H$. This means precisely that

$$
\mathcal{E}^{\prime}(u) v=\langle\nabla \mathcal{E}(u), v\rangle \text { for all } v \in V
$$

i.e. $\mathcal{E}^{\prime}(u) \in V^{*}=\mathcal{L}(V, \mathbb{R})$ extends to $\overline{\mathcal{E}^{\prime}(u)} \in \mathcal{L}(H, \mathbb{R})$ via $(15)$. Thus, $\overline{\mathcal{E}^{\prime}(u)}=\nabla \mathcal{E}(u)$ under the isomorphism $H \cong H^{*}$ given by the Riesz-Fréchet Theorem.

Corollary 5.2. Let $V$ be a Hilbert space, $U \subset V$ be an open set, $m \in \mathbb{N}$ and let $\mathcal{E} \in \mathcal{C}^{\omega}(U ; \mathbb{R}), \mathcal{G} \in \mathcal{C}^{\omega}\left(U ; \mathbb{R}^{m}\right)$. Let $\bar{u} \in U$ and suppose that

(i) there exists a Hilbert space $(H,\langle\cdot, \cdot\rangle)$ with $V \hookrightarrow H$ densely,

(ii) $\mathcal{E}$ possesses an $H$-gradient $\nabla \mathcal{E}(u)$ at each $u \in U$ and the map $u \mapsto \nabla \mathcal{E}(u): U \rightarrow H$ is analytic,

(iii) the second derivative $\mathcal{E}^{\prime \prime}(\bar{u})=(\nabla \mathcal{E})^{\prime}(\bar{u}): V \rightarrow H$ is Fredholm of index zero 1

(iv) for any $u \in U$, the components $\mathcal{G}_{k}: U \rightarrow \mathbb{R}$ of $\mathcal{G}$ possess $H$-gradients $\nabla \mathcal{G}_{k}$ such that $U \ni u \mapsto \nabla \mathcal{G}_{k}(u) \in H$ is analytic for all $k=1, \ldots, m$,

(v) the Fréchet derivatives $\left(\nabla \mathcal{G}_{k}\right)^{\prime}(\bar{u}): V \rightarrow H$ are compact for all $k=1, \ldots, m$,

(vi) $\mathcal{G}(\bar{u})=0$ and the $H$-gradients $\nabla \mathcal{G}_{1}(\bar{u}), \ldots, \nabla \mathcal{G}_{m}(\bar{u})$ are linearly independent.

Then, $\mathcal{M}:=\{u \in U \mid \mathcal{G}(u)=0\}$ is locally an analytic submanifold of $V$ of codimension $m$ near $\bar{u}$.

If $\bar{u}$ is a critical point of $\left.\mathcal{E}\right|_{\mathcal{M}}$, then the restriction satisfies a refined Eojasiewicz-Simon gradient inequality at $\bar{u}$, i.e. there exist $C, \sigma>0$ and $\theta \in\left(0, \frac{1}{2}\right]$ such that for any $u \in \mathcal{M}$ with $\|u-\bar{u}\|_{V} \leq \sigma$, we have

$$
|\mathcal{E}(u)-\mathcal{E}(\bar{u})|^{1-\theta} \leq C\|P(u) \nabla \mathcal{E}(u)\|_{H},
$$

where $P(u): H \rightarrow H$ is the orthogonal projection onto $\overline{\mathcal{T}_{u} \mathcal{M}}:=\overline{\mathcal{T}_{u} \mathcal{M}} \|^{\|\cdot\|_{H}}$.

Remark 5.3. Requiring $V$ to be a Hilbert space in Corollary 5.2 is no additional assumption. Indeed, if hypothesis (iii) in Corollary 5.2 is satisfied for $V$ merely a Banach space, $\mathcal{E}^{\prime \prime}(\bar{u}): V \rightarrow H$ is a compact perturbation of an isomorphism by [4. Theorem 7.10]. In particular, $V$ and $H$ are isomorphic, so $V$ has to be a Hilbert space.

Remark 5.4. In the case $m=1$ in Corollary 5.2, the projection $P(u) \in \mathcal{L}(H)$ onto $\overline{\mathcal{T}_{u} \mathcal{M}}$ is given by $P(u) y=y-\frac{\langle\nabla \mathcal{G}(u), y\rangle}{\|\nabla \mathcal{G}(u)\|_{H}^{2}} \nabla \mathcal{G}(u)$, where $\nabla \mathcal{G}:=\nabla \mathcal{G}_{1}$. This yields

$$
|\mathcal{E}(u)-\mathcal{E}(\bar{u})|^{1-\theta} \leq C\left\|\nabla \mathcal{E}(u)-\frac{\langle\nabla \mathcal{G}(u), \nabla \mathcal{E}(u)\rangle}{\|\nabla \mathcal{G}(u)\|_{H}^{2}} \nabla \mathcal{G}(u)\right\|_{H} .
$$

The scalar $\lambda(u):=\frac{\langle\nabla \mathcal{G}(u), \nabla \mathcal{E}(u)\rangle}{\|\nabla \mathcal{G}(u)\|^{2}}$ is often referred to as the Langrange multiplier, since if the right hand side of (17) is zero, $\lambda(u)$ is exactly the Lagrange multiplier for the function $\mathcal{E}$ subject to the constraint $\mathcal{G}(u)=0$ (cf. [16, Chapter 2]).

\footnotetext{
${ }^{1}$ The equation $\mathcal{E}^{\prime \prime}(\bar{u})=(\nabla \mathcal{E})^{\prime}(u)$ has to be understood in the sense of the identification $\mathcal{E}^{\prime}(u)=\nabla \mathcal{E}(u)$, cf. Definition and Lemma 5.1
} 
The following shows that assumption (vi) in Corollary 5.2 is just the equivalent formulation of hypothesis (vi) in Theorem 1.4.

Lemma 5.5. Let $V$ be a Banach space and let $(H,\langle\cdot, \cdot\rangle)$ be a Hilbert space such that $V \hookrightarrow H$ densely. Let $U \subset V$ open, $u \in U$ and suppose $\mathcal{G} \in \mathcal{C}^{1}\left(U ; \mathbb{R}^{m}\right)$ possesses $H$ gradients $\nabla \mathcal{G}_{1}(u), \ldots, \nabla \mathcal{G}_{m}(u)$ in the sense of Definition and Lemma 5.1. Then the following are equivalent.

(i) $\mathcal{G}^{\prime}(u): V \rightarrow \mathbb{R}^{m}$ is surjective,

(ii) $\nabla \mathcal{G}_{1}(u), \ldots, \nabla \mathcal{G}_{m}(u)$ are linearly independent in $H$.

Proof. Assume (i) holds and let $\lambda \in \mathbb{R}^{m}$ be such that $\sum_{k=1}^{m} \lambda_{k} \nabla \mathcal{G}_{k}(u)=0$ in $H$. Then, for any $v \in V \subset H$ we have $0=\sum_{k=1}^{m} \lambda_{k}\left\langle\nabla \mathcal{G}_{k}(u), v\right\rangle=\left\langle\lambda, \mathcal{G}^{\prime}(u) v\right\rangle_{\mathbb{R}^{m}}$ by Definition and Lemma 5.1. Hence, $\lambda \in\left(\operatorname{Im} \mathcal{G}^{\prime}(u)\right)^{\perp_{\mathbb{R}^{m}}}=\{0\}$ by (i). Conversely, suppose (ii) holds and $\lambda \in\left(\operatorname{Im} \mathcal{G}^{\prime}(u)\right)^{\perp_{\mathbb{R}}^{m}}$. Then, we have

$$
0=\left\langle\lambda, \mathcal{G}^{\prime}(u) v\right\rangle_{\mathbb{R}^{m}}=\sum_{k=1}^{m} \lambda_{k}\left\langle\nabla \mathcal{G}_{k}(u), v\right\rangle=\left\langle\sum_{k=1}^{m} \lambda_{k} \nabla \mathcal{G}_{k}(u), v\right\rangle \text { for all } v \in V .
$$

As a consequence, $\sum_{k=1}^{m} \lambda_{k} \nabla \mathcal{G}_{k}(u)=0$ in $H$ by density of $V \subset H$, thus $\lambda=0$ by (ii).

Proof of Corollary 5.2. Assumptions (i)-(iv) of Theorem 1.4 are satisfied if we choose $Y=Y^{*}=H$ under the identification of $H$ with its image in $V^{*}$. Note that the extension of $\mathcal{G}^{\prime}(u)$ is given by

$$
\overline{\mathcal{G}^{\prime}(u)} y=\left(\left\langle\nabla \mathcal{G}_{1}(u), y\right\rangle, \ldots,\left\langle\nabla \mathcal{G}_{m}(u), y\right\rangle\right)^{T} \text { for } y \in H .
$$

Thus, assumption (v) of Theorem 1.4 is satisfied if and only if $\left(\nabla \mathcal{G}_{k}\right)^{\prime}(\bar{u}): V \rightarrow H$ is compact for all $k=1, \ldots, m$ which is exactly assumption (v) in Corollary [5.2. By Lemma 5.5. assumption (vi) in Theorem 1.4 is also satisfied. We conclude that there exists $C, \sigma>0$ and $\theta \in\left(0, \frac{1}{2}\right]$ such that for any $u \in \mathcal{M}$ with $\|u-\bar{u}\|_{V} \leq \sigma$, we have

$$
|\mathcal{E}(u)-\mathcal{E}(\bar{u})|^{1-\theta} \leq C\left\|\mathcal{E}^{\prime}(u)\right\|_{\overline{\mathcal{T}}_{u} \mathcal{M}^{*}} .
$$

By Proposition 3.3, $\overline{\mathcal{T}_{u} \mathcal{M}}=\operatorname{ker} \overline{\mathcal{G}^{\prime}(u)}$. Consequently, by (18), we have

$$
\overline{\mathcal{T}_{u} \mathcal{M}}=\left\{y \in H \mid\left\langle\nabla \mathcal{G}_{k}(u), y\right\rangle=0 \text { for all } k=1, \ldots, m\right\} .
$$

Hence, if $P(u) \in \mathcal{L}(H)$ denotes the orthogonal projection onto $\overline{\mathcal{T}_{u} \mathcal{M}} \subset H$ we can estimate the right hand side of (19) by

$$
\begin{aligned}
& \left\|\mathcal{E}^{\prime}(u)\right\|_{\overline{\mathcal{T}}_{u} \mathcal{M}^{*}}=\sup _{0 \neq y \in \overline{\mathcal{T}_{u} \mathcal{M}}} \frac{\mathcal{E}^{\prime}(u) y}{\|y\|_{H}}=\sup _{0 \neq y \in \overline{\mathcal{T}_{u} \mathcal{M}}} \frac{\langle\nabla \mathcal{E}(u), P(u) y\rangle}{\|y\|_{H}} \\
& =\sup _{0 \neq y \in \overline{\mathcal{T}_{u} \mathcal{M}}} \frac{\langle P(u) \nabla \mathcal{E}(u), y\rangle}{\|y\|_{H}} \leq\|P(u) \nabla \mathcal{E}(u)\|_{H} .
\end{aligned}
$$

Together with (19), this proves (16). 
In the setting of Corollary 5.2 , we may deduce the following abstract convergence result for the associated gradient flow.

Corollary 5.6. Let $\mathcal{E}, \mathcal{G}$ be as in Corollary 5 .2. with $m=1$ and suppose $u \in \mathcal{C}^{1}([0, \infty) ; V)$ is a solution of the constrained gradient flow equation

$$
\left\{\begin{array}{l}
\partial_{t} u=-\nabla \mathcal{E}(u)+\lambda(u) \nabla \mathcal{G}(u), \quad t>0 \\
u(0)=u_{0},
\end{array},\right.
$$

where $\lambda(u)$ is as in (17). Assume that $\overline{\{u(t) \mid t \geq 0\}} \subset V$ is compact. Then $\lim _{t \rightarrow \infty} u(t)$ exists in $V$.

Remark 5.7. The key idea in the proof of Corollary[5.6 is the following (formal) computation, based on [33]. If $\left.\mathcal{E}\right|_{\mathcal{G}^{-1}\{0\}}$ satisfies a refined Lojasiewicz-Simon gradient inequality near $\bar{u} \in \overline{\{u(t) \mid t \geq 0\}}$, then

$$
\begin{aligned}
-\frac{\mathrm{d}}{\mathrm{d} t}(\mathcal{E}(u)-\mathcal{E}(\bar{u}))^{\theta} & =-\theta(\mathcal{E}(u)-\mathcal{E}(\bar{u}))^{\theta-1}\left\langle\nabla \mathcal{E}(u), \partial_{t} u\right\rangle \\
& =\theta(\mathcal{E}(u)-\mathcal{E}(\bar{u}))^{\theta-1}\|\nabla \mathcal{E}(u)-\lambda(u) \nabla \mathcal{G}(u)\|_{H}\left\|\partial_{t} u\right\|_{H} \\
& \geq \frac{\theta}{C}\left\|\partial_{t} u\right\|_{H} .
\end{aligned}
$$

This implies $\partial_{t} u \in L^{1}([0, \infty) ; H)$ which yields the claim (see [11, Theorem 12.2] for a detailed presentation of this argument, with weaker regularity assumptions).

\section{Optimality discussion}

In this section, we will discuss why the assumptions in Theorem 1.4 and Corollary 5.2 cannot be omitted.

First, we provide an example, inspired by the Hilbert space case in [19, Theorem 2.1], which implies that in any Banach space of infinite dimension, there will exist an energy which fails to satisfy the Eojasiewicz-Simon gradient inequality. The construction relies on the following nontrivial fact.

Theorem 6.1. Let $V$ be a Banach space of infinite dimension and let $\varepsilon>0$. Then there exist sequences $\left(e_{n}\right)_{n \in \mathbb{N}} \subset V$ with $\left\|e_{n}\right\|=1$ for all $n \in \mathbb{N}$ and $\left(\phi_{k}\right)_{k \in \mathbb{N}} \subset V^{*}$ with $\left\|\phi_{k}\right\| \leq 2(1+\varepsilon)$ for all $k \in \mathbb{N}$ such that

$$
\phi_{k}\left(e_{n}\right)=\delta_{k, n}, \quad \text { for all } k, n \in \mathbb{N} .
$$

Proof. See Appendix B.

Example 6.2. Let $V$ be a Banach space of infinite dimension and $\varepsilon>0$. Let $\left(e_{n}\right)_{n \in N}$ and $\left(\phi_{k}\right)_{k \in \mathbb{N}}$ be as in Theorem 6.1. Let $\lambda \in \ell^{1}(\mathbb{N})$ with $\lambda_{k} \neq 0$ for all $k \in \mathbb{N}$. Then, $x=0$ is a critical point of the analytic energy

$$
\mathcal{E}(x):=\frac{1}{2} \sum_{k=1}^{\infty} \lambda_{k}\left|\phi_{k}(x)\right|^{2}, \text { for } x \in V,
$$

but $\mathcal{E}$ satisfies no Lojasiewicz-Simon gradient inequality around $x=0$. 
Proof. First, we will prove that $\mathcal{E}$ is analytic. Indeed, we have $\mathcal{E}(x)=\Phi(x, x)$, where $\Phi(x, y):=\sum_{k=1}^{\infty} \lambda_{k} \phi_{k}(x) \phi_{k}(y)$ for $x, y \in V$. Note that $\Phi: V \times V \rightarrow \mathbb{R}$ is bilinear and bounded by

$$
|\Phi(x, y)| \leq \beta^{2} \sum_{k=1}^{\infty}\left|\lambda_{k}\right|\|x\|\|y\| \text { for all } x, y \in V,
$$

where $\beta:=2(1+\varepsilon)$. By Example 2.3 , $\Phi$ and hence $\mathcal{E}$ is analytic. Furthermore, we find

$$
\mathcal{E}^{\prime}(x)(y)=\sum_{k=1}^{\infty} \lambda_{k} \phi_{k}(x) \phi_{k}(y) \text { for all } x, y \in V .
$$

Clearly, $\mathcal{E}^{\prime}(0)=0$. For $k \in \mathbb{N}$ and $t>0$ we have

$$
\left\|\mathcal{E}^{\prime}\left(t e_{n}\right)\right\|_{V^{*}}=\sup _{\|y\|_{V}=1} \sum_{k=1}^{\infty} \lambda_{k} \phi_{k}\left(t e_{n}\right) \phi_{k}(y) \leq t\left|\lambda_{n}\right|\left\|\phi_{n}\right\|_{V^{*}} \leq \beta t\left|\lambda_{n}\right|
$$

and $\mathcal{E}\left(t e_{n}\right)=\frac{1}{2} \lambda_{n} t^{2}$. Thus, if $\mathcal{E}$ satisfied a Eojasiewicz-Simon gradient inequality for some $C, \sigma, \theta>0$, for all $n \in \mathbb{N}$ and $0<t \leq \sigma$ we would get

$$
\left(\frac{1}{2}\left|\lambda_{n}\right| t^{2}\right)^{1-\theta} \leq C \beta\left|\lambda_{n}\right| t
$$

Dividing by $\left|\lambda_{n}\right|$ and letting $n \rightarrow \infty$ yields a contradiction, since $\lambda_{n} \rightarrow 0$ as $\lambda \in \ell^{1}(\mathbb{N})$.

It is not too difficult to see that the second derivative of $\mathcal{E}$ in Example 6.2 fails to be Fredholm. Consequently, condition (iii) in Theorem 1.2 (and Theorem 1.4) is violated, whereas conditions (i)-(ii) are satisfied with $Z=H(Y=H$, respectively), indicating that mere analyticity of the energy is not enough. In fact, the following result shows that it is never sufficient in infinite dimensions.

Corollary 6.3. Let $V$ be a Banach space, and let $U \subset V$ be open. Then, $\operatorname{dim} V<\infty$ if and only if every analytic function $\mathcal{E} \in \mathcal{C}^{\omega}(U ; \mathbb{R})$ satisfies a tojasiewicz-Simon gradient inequality at each of its critical points.

For the sake of simplicity, throughout the rest of this section we restrict ourselves to the Hilbert space case in Corollary 5.2 with $V=H$. That way, assumptions (i), (ii) and (iv) are automatically satisfied if the energy and the constraint are analytic. The next example shows that we can not drop the compactness assumption (v) in Corollary 5.2 .

Example 6.4. Consider the Hilbert space $H=\mathbb{R} \times \ell^{2}(\mathbb{N})$ and let $\lambda \in \ell^{1}(\mathbb{N})$. We write elements $x \in H$ as $x=\left(x_{0}, x^{\prime}\right)$ with $x^{\prime} \in \ell^{2}(\mathbb{N})$. The natural norm on $H$ is given by $\|x\|_{H}^{2}:=\left|x_{0}\right|^{2}+\left\|x^{\prime}\right\|_{\ell^{2}(\mathbb{N})}^{2}$. For $x=\left(x_{0}, x^{\prime}\right) \in H$ define

$$
\mathcal{E}(x):=x_{0}+\sum_{n=1}^{\infty}\left|x_{n}^{\prime}\right|^{2} \text { for } x \in H
$$


Then $\mathcal{E}$ satisfies assumptions (i)-(iii) in Corollary 5.2 with $V=H$. We define

$$
\psi: \ell^{2}(\mathbb{N}) \rightarrow \mathbb{R}, \psi\left(x^{\prime}\right):=\sum_{n=1}^{\infty}\left(\lambda_{n}-1\right)\left|x_{n}^{\prime}\right|^{2}
$$

and consider $\mathcal{G}: H \rightarrow \mathbb{R}, \mathcal{G}(x):=x_{0}-\psi\left(x^{\prime}\right)$ and $\mathcal{M}:=\mathcal{G}^{-1}(\{0\})$. Then, $\left.\mathcal{E}\right|_{\mathcal{M}}$ does not satisfy a refined Eojasiewicz-Simon gradient inequality at the origin, but satisfies all assumptions of Corollary 5.2 with $V=H$ except assumption (v).

Proof. It is easy to see that $\mathcal{E}, \psi$ and $\mathcal{G}$ are analytic. Given $\bar{x}, y \in H$, a short computation yields $(\nabla \mathcal{E})^{\prime}(\bar{x}) y=\left(0,2 y^{\prime}\right)$, so the second derivative is Fredholm with index zero and the first part of the statement is proven.

Moreover, $\mathcal{G}$ possesses an $H$-gradient $\nabla \mathcal{G}\left(x_{0}, x^{\prime}\right)=\left(1,\left(2\left(\lambda_{n}-1\right) x_{n}^{\prime}\right)_{n \in \mathbb{N}}\right) \in H$ and the gradient map is analytic. Also note that $\mathcal{G}(0)=0$ and $\nabla \mathcal{G}(0)=(1,0)$, so assumptions (iv) and (vi) of Corollary 5.2 are satisfied. By Example $2.10, \mathcal{M}$ is an analytic submanifold of $H$ near the origin, with a single chart $\varphi: \ell^{2}(\mathbb{N}) \rightarrow H, \varphi\left(x^{\prime}\right):=\left(\psi\left(x^{\prime}\right), x^{\prime}\right) \in H$ which coincides with the chart from Theorem 3.1 in this example. However, note that the operator $T:=(\nabla \mathcal{G})^{\prime}(0): H \rightarrow H,(\nabla \mathcal{G})^{\prime}(0)\left(x_{0}, x^{\prime}\right)=\left(0,\left(2\left(\lambda_{n}-1\right) x_{n}^{\prime}\right)_{n \in \mathbb{N}}\right)$ is not compact. Indeed, let $x_{k}:=\left(0, e_{k}^{\prime}\right) \in H$ where $e_{k}^{\prime} \in \ell^{2}(\mathbb{N})$ is the standard $k$-th unit vector. A short computation yields $T x_{k} \rightarrow 0$, however $T x_{k} \not \rightarrow 0$, since $\left\|T x_{k}\right\|_{H}=2\left|\lambda_{k}-1\right| \rightarrow 1$, so $T$ cannot be compact. For $x^{\prime} \in \ell^{2}(\mathbb{N})$ we have

$$
(\mathcal{E} \circ \varphi)\left(x^{\prime}\right)=\psi\left(x^{\prime}\right)+\sum_{n=1}^{\infty}\left|x_{n}^{\prime}\right|^{2}=\sum_{n=1}^{\infty} \lambda_{n}\left|x_{n}^{\prime}\right|^{2} .
$$

Similar to Example 6.2, one can show that $\mathcal{E} \circ \varphi$ does not satisfy a Lojasiewicz-Simon gradient inequality at the origin $x^{\prime}=0$ by assuming the inequality holds and then testing it with $x^{\prime}=e_{k}^{\prime} \in \ell^{2}(\mathbb{N})$ for all $k \in \mathbb{N}$. Lemma 4.2 then implies that $\left.\mathcal{E}\right|_{\mathcal{M}}$ cannot satisfy a refined Łojasiewicz-Simon gradient inequality at $x=0$ either 2

Example 6.4 shows that assumption (v) in Corollary 5.2 cannot be omitted. Note that while one can easily show using Theorem 1.2 that $\mathcal{E}$ as in Example 6.4 satisfies a Łojasiewicz-Simon gradient inequality, $\left.\mathcal{E}\right|_{\mathcal{M}}$ does not satisfy the refined inequality (3). In particular, the property of satisfying a Łojasiewicz-Simon gradient inequality does in general not behave well under the restriction to a submanifold, even if we assume finite codimension.

Let us also remark that condition (vi) in Corollary 5.2 is in general necessary to guarantee that $\mathcal{M}$ is a manifold. Finally, note that our main result only considers submanifolds of finite codimension. The following example shows that our main result cannot be extended to the case of infinite codimension, even for linear subspaces.

Example 6.5. Consider the Hilbert space $H:=\ell^{2}(\mathbb{N}) \times \ell^{2}(\mathbb{N})$ with

$$
\left\langle\left(x, x^{\prime}\right),\left(y, y^{\prime}\right)\right\rangle_{H}:=\langle x, y\rangle_{\ell^{2}(\mathbb{N})}+\left\langle x^{\prime}, y^{\prime}\right\rangle_{\ell^{2}(\mathbb{N})}, \text { for }\left(x, x^{\prime}\right),\left(y, y^{\prime}\right) \in H .
$$

\footnotetext{
${ }^{2}$ At this point it is crucial that in Lemma 4.2 we did not require assumption (v) of Theorem 1.4 to be satisfied.
} 
Then, the energy $\mathcal{E}: H \rightarrow \mathbb{R}, \mathcal{E}\left(x, x^{\prime}\right):=\sum_{n=1}^{\infty}\left|x_{n}\right|^{2}-\left|x_{n}^{\prime}\right|^{2}$ satisfies assumptions (i)-(iii) in Corollary 5.2 with $V=H$. For the constraint function $\mathcal{G}: H \rightarrow \ell^{2}(\mathbb{N}),\left(x, x^{\prime}\right) \mapsto\left(x_{n}-\right.$ $\left.\sqrt{1+n^{-2}} x_{n}^{\prime}\right)_{n \in \mathbb{N}}$, the set $\mathcal{M}:=\mathcal{G}^{-1}(\{0\})$ is a linear subspace of $H$ and $\left(x, x^{\prime}\right)=(0,0)$ is a constrained critical point of $\left.\mathcal{E}\right|_{\mathcal{M}}$. Moreover, $\mathcal{G}$ and $\mathcal{E}$ are analytic, $\nabla \mathcal{G}(0,0) \neq 0$ and $0=(\nabla \mathcal{G})^{\prime}(0,0): H \rightarrow H$ is compact. However, $\left.\mathcal{E}\right|_{\mathcal{M}}$ does not satisfy a refined Eojasiewicz-Simon gradient inequality near the origin.

Proof. The properties of $\mathcal{E}$ and $\mathcal{G}$ can be shown as in in Example 6.4. The natural chart for $\mathcal{M}$ is given by

$$
\varphi: \ell^{2}(\mathbb{N}) \rightarrow H, \varphi(y):=\left(\left(\sqrt{1+n^{-2}} y_{n}\right)_{n \in \mathbb{N}},\left(y_{n}\right)_{n \in \mathbb{N}}\right)
$$

and we observe that $(\mathcal{E} \circ \varphi)(y)=\sum_{n=1}^{\infty} \frac{1}{n^{2}}\left|y_{n}\right|^{2}$ cannot satisfy a Łojasiewicz-Simon gradient inequality near $x=0$ by Example 6.2. Hence, by an argument similar to Lemma 4.2, neither does $\left.\mathcal{E}\right|_{\mathcal{M}}$.

\section{Applications}

In this section, we will apply our result from Theorem 1.4 to different energies on Sobolev spaces with isoperimetric constraints (cf. [16, Chapter 2.1]). Like in Corollary [5.6 this can be then used to conclude convergence for precompact solutions of the associated gradient flows.

\subsection{Surface area with an isoperimetric constraint}

Throughout this subsection, we assume that $\Omega \subset \mathbb{R}^{d}$ is a domain with $\mathcal{C}^{1,1}$-boundary. We want to study the surface area or $d$-dimensional Hausdorff measure of $\operatorname{graph}(u) \subset \mathbb{R}^{d+1}$ given by

$$
\mathcal{E}(u):=\int_{\Omega} \sqrt{1+|\nabla u|^{2}} \mathrm{~d} x .
$$

Note that while this energy is already defined if we merely require $u \in W^{1,1}(\Omega)$, a natural space to study a $L^{2}$-gradient flow would be $W^{2,2}(\Omega)$. However, we consider $u \in V:=W^{2, p}(\Omega) \cap W_{0}^{1, p}(\Omega)$ with $d<p<\infty$ and $Y:=L^{q}(\Omega)$ where $\frac{1}{p}+\frac{1}{q}=1$. The condition on $p$ and our choice of spaces will imply analyticity (cf. Remark 1.7). We want to study $\mathcal{E}$ on the set of functions which satisfy the constraint

$$
\mathcal{G}(u)=\int_{\Omega} g(u) \mathrm{d} x=\int_{\Omega} g(u(x)) \mathrm{d} x=0,
$$

where $g: \mathbb{R} \rightarrow \mathbb{R}$ is an analytic function. Note that the energy as well as the constraint are well defined since $W^{2, p}(\Omega)$ embeds into both $W^{1,1}(\Omega)$ and $\mathcal{C}(\bar{\Omega})$ by [17, Corollary 7.11]. Moreover, $V \hookrightarrow L^{q}(\Omega)$ densely, so we get an induced embedding $L^{p}(\Omega)=Y^{*} \hookrightarrow V^{*}$. We recall the following important property of Nemytskii operators. 
Theorem 7.1 ([5, Theorem 6.8]). Let $F \in \mathcal{C}(\mathbb{R})$. Then, the superposition operator $\mathcal{F}: \mathcal{C}(\bar{\Omega}) \rightarrow \mathcal{C}(\bar{\Omega}), \mathcal{F}(v)=F(v)$ is analytic if and only if the function $F$ is.

Lemma 7.2. The map $\mathcal{G}: V \rightarrow \mathbb{R}$ is analytic with

$$
\mathcal{G}^{\prime}(u) v=\int_{\Omega} g^{\prime}(u) v \mathrm{~d} x, \text { for } u, v \in V .
$$

In particular, $\mathcal{G}^{\prime}(u)=g^{\prime}(u) \in Y^{*}=L^{p}(\Omega)$.

Proof. By the embedding $W^{2, p}(\Omega) \hookrightarrow \mathcal{C}(\bar{\Omega})$, the map $V \ni u \mapsto u \in \mathcal{C}(\bar{\Omega})$ is analytic. Hence, so is $V \ni u \mapsto g(u) \in \mathcal{C}(\bar{\Omega})$ by Theorem 7.1. Integrating is analytic by Example 2.3, since it is linear and bounded. Using Theorem 2.2, this yields $\mathcal{G} \in \mathcal{C}^{\omega}(V ; \mathbb{R})$. Furthermore, (22) follows, since for $u, v \in V$, we have

$$
\mathcal{G}^{\prime}(u) v=\left.\frac{\mathrm{d}}{\mathrm{d} t}\right|_{t=0} \mathcal{G}(u+t v)=\left.\int_{\Omega} \frac{\mathrm{d}}{\mathrm{d} t}\right|_{t=0} g(u+t v) \mathrm{d} x=\int_{\Omega} g^{\prime}(u) v \mathrm{~d} x .
$$

Clearly, $\mathcal{G}^{\prime}(u)=g^{\prime}(u) \in Y^{*}=L^{p}(\Omega)$, since $g^{\prime}(u) \in \mathcal{C}(\bar{\Omega}) \subset L^{p}(\Omega)$. Moreover, we have $\mathcal{G}^{\prime} \in \mathcal{C}^{\omega}\left(V, Y^{*}\right)$, since $g^{\prime}: \mathbb{R} \rightarrow \mathbb{R}$ is analytic and so is $V \ni u \mapsto g^{\prime}(u) \in L^{p}(\Omega)$ by the embeddings $V \hookrightarrow \mathcal{C}(\bar{\Omega})$ and $\mathcal{C}(\bar{\Omega}) \hookrightarrow L^{p}(\Omega)$ and using Theorems 2.2 and 7.1 ,

As a next step, we compute the second derivative of $\mathcal{G}$.

Lemma 7.3. The operator $\mathcal{G}^{\prime \prime}(u): V \rightarrow Y^{*}$ is compact for any $u \in V$.

Proof. Let $u, v \in V$. From (22), we conclude

$$
\mathcal{G}^{\prime \prime}(u) v=\left.\frac{\mathrm{d}}{\mathrm{d} t}\right|_{t=0} g^{\prime}(u+t v)=g^{\prime \prime}(u) v \in L^{p}(\Omega) .
$$

Since $u \in V \hookrightarrow \mathcal{C}(\bar{\Omega}), g^{\prime \prime}(u) \in \mathcal{C}(\bar{\Omega})$ and hence $V \ni v \mapsto g^{\prime \prime}(u) v \in Y^{*}=L^{p}(\Omega)$ is compact, since the embedding $V \ni v \mapsto v \in L^{p}(\Omega)$ is compact. It follows that $\mathcal{G}^{\prime \prime}(u): V \rightarrow Y^{*}$ is compact.

Lemma 7.4. The map $\mathcal{E}: V \rightarrow \mathbb{R}$ is analytic with

$$
\mathcal{E}^{\prime}(u) v=-\int_{\Omega} \operatorname{div}\left(\frac{\nabla u}{\sqrt{1+|\nabla u|^{2}}}\right) v \mathrm{~d} x, \text { for } u, v \in V .
$$

Moreover, $\mathcal{E}^{\prime}(u) \in Y^{*}=L^{p}(\Omega)$ for all $u \in V$.

This analyticity statement motivates our choice of spaces in the beginning of this section.

Proof. We first note that the following maps are analytic.

(i) The embedding $i: V \hookrightarrow \mathcal{C}^{1}(\bar{\Omega})$ since $d>p$ using Example 2.3 , 
(ii) The map $\mathcal{C}^{1}(\bar{\Omega}) \rightarrow \mathcal{C}(\bar{\Omega}), u \mapsto|\nabla u|^{2}$ by Example 2.3, since it is the diagonal of a bounded bilinear map.

(iii) The map $\mathcal{C}(\bar{\Omega} ;(-1, \infty)) \subset \mathcal{C}(\bar{\Omega}) \rightarrow \mathcal{C}(\bar{\Omega}), \mathcal{F}(v)=(1+v)^{-\alpha}$ for $\alpha>0$ by Theorem 7.1, since the map $F:(-1, \infty) \rightarrow \mathbb{R}, F(x)=(1+x)^{-\alpha}$ is analytic.

(iv) The map

$$
V \rightarrow \mathcal{C}(\bar{\Omega}), u \mapsto\left(1+|\nabla u|^{2}\right)^{-\alpha}
$$

for $\alpha>0$ as a composition of the maps in (i)-(iii) using Theorem 2.2 .

(v) The map $\mathcal{C}(\bar{\Omega}) \rightarrow \mathbb{R}, v \mapsto \int_{\Omega} v \mathrm{~d} x$ by Example 2.3, since it is linear and bounded.

Since $\mathcal{E}$ can be written as the composition of these maps, $\mathcal{E}$ is analytic. For a proof of (23), consider [16, Chapter 1, 2.2 Example 5]. Note that $\mathcal{E}^{\prime}(u) \in L^{p}(\Omega)$, since for $u \in W^{2, p}(\Omega)$, we have using summation convention

$$
\operatorname{div}\left(\frac{\nabla u}{\sqrt{1+|\nabla u|^{2}}}\right)=\frac{\Delta u}{\sqrt{1+|\nabla u|^{2}}}-\partial_{i} u \frac{\partial_{i} \partial_{j} u \partial_{j} u}{\left(1+|\nabla u|^{2}\right)^{\frac{3}{2}}} .
$$

By the embedding $W^{2, p}(\Omega) \hookrightarrow \mathcal{C}^{1}(\bar{\Omega})$ and since the denominators are bounded from below, we conclude that $\mathcal{E}^{\prime}(u) \in L^{p}(\Omega)$ for $u \in W^{2, p}(\Omega)$.

Lemma 7.5. The function $\mathcal{E}^{\prime}: V \rightarrow L^{p}(\Omega), u \mapsto \mathcal{E}^{\prime}(u)$ is analytic.

Proof. The following maps are analytic.

(i) The map $V \rightarrow L^{p}(\Omega), u \mapsto \partial_{i} \partial_{j} u$ for any $i, j \in\{1, \ldots, d\}$ by Example 2.3.

(ii) The maps $u \mapsto\left(1+|\nabla u|^{2}\right)^{-\frac{1}{2}}, u \mapsto\left(1+|\nabla u|^{2}\right)^{-\frac{3}{2}}: V \rightarrow \mathcal{C}(\bar{\Omega})$ by (24).

(iii) $V \rightarrow \mathcal{C}(\bar{\Omega}), u \mapsto \partial_{j} u$ for any $j \in\{1, \ldots, d\}$ by Example 2.3 .

Since the pointwise multiplications $\mathcal{C}(\bar{\Omega}) \times L^{p}(\Omega) \rightarrow L^{p}(\Omega)$ and $\mathcal{C}(\bar{\Omega}) \times \mathcal{C}(\bar{\Omega}) \rightarrow \mathcal{C}(\bar{\Omega})$ are bilinear and bounded, they are analytic by Example 2.3. Hence, so is $u \mapsto \mathcal{E}^{\prime}(u)$ by (25) and Theorem 2.2 .

Lemma 7.6. Let $u \in V$. Then the Fréchet derivative $\mathcal{E}^{\prime \prime}(u): V \rightarrow Y^{*}$ is Fredholm of index zero.

Proof. By Lemma 7.5, for $u, v \in V$, we compute

$$
\begin{aligned}
\mathcal{E}^{\prime \prime}(u) v & =\left.\frac{\mathrm{d}}{\mathrm{d} t}\right|_{t=0} \mathcal{E}^{\prime}(u+t v)=-\operatorname{div}\left(\frac{\nabla v \sqrt{1+|\nabla u|^{2}}-\nabla u \frac{\langle\nabla u, \nabla v\rangle}{\sqrt{1+|\nabla u|^{2}}}}{1+|\nabla u|^{2}}\right) \\
& =-\frac{\Delta v}{\sqrt{1+|\nabla u|^{2}}}+\frac{1}{\left(1+|\nabla u|^{2}\right)^{\frac{3}{2}}}\langle\nabla u, \nabla\langle\nabla u, \nabla v\rangle\rangle+\tilde{K} v
\end{aligned}
$$




$$
=-\frac{1}{\left(1+|\nabla u|^{2}\right)^{\frac{3}{2}}}\left(\delta_{i j}\left(1+|\nabla u|^{2}\right)-\partial_{i} u \partial_{j} u\right) \partial_{i} \partial_{j} v+K v=:-A v+K v
$$

using summation convention, where $\tilde{K}, K: V \rightarrow L^{p}(\Omega)$ only contain terms in $v$ of order 1 or lower, whence are compact by the Rellich-Kondrachov Theorem [17, Theorem 7.26]. It is easy to see that $A$ uniformly is elliptic, hence $A: W^{2, p}(\Omega) \cap W_{0}^{1, p}(\Omega) \rightarrow L^{p}(\Omega)$ is an isomorphism by [17, Theorem 9.15]. Therefore, $\mathcal{E}^{\prime \prime}(u)=-A+K: V \rightarrow Y^{*}$ is Fredholm of index zero by Proposition 2.5

Now, we can apply Theorem 1.4 to our situation.

Theorem 7.7. Let $\bar{u} \in V, \mathcal{G}(\bar{u})=0$ with $g^{\prime}(\bar{u}) \not \equiv 0$ be a constraint critical point of $\mathcal{E}$ on $\mathcal{M}=\{u \in V \mid \mathcal{G}(u)=0\}$. Then, $\mathcal{M}$ is locally a manifold near $\bar{u}$ and satisfies a Eojasiewicz-Simon gradient inequality on $\mathcal{M}$, i.e. there exist $C, \sigma>0$ and $\theta \in\left(0, \frac{1}{2}\right]$ such that for any $u \in \mathcal{M}$ with $\|u-\bar{u}\|_{W^{2, p}(\Omega)} \leq \sigma$, we have

$$
|\mathcal{E}(u)-\mathcal{E}(\bar{u})|^{1-\theta} \leq C\left\|\mathcal{E}^{\prime}(u)-\frac{\int_{\Omega} \mathcal{E}^{\prime}(u) g^{\prime}(u) \mathrm{d} x}{\int_{\Omega}\left(g^{\prime}(u)\right)^{2} \mathrm{~d} x} g^{\prime}(u)\right\|_{L^{p}(\Omega)} .
$$

Proof. We verify that Theorem 1.4 is applicable with $U=V=W^{2, p}(\Omega) \cap W_{0}^{1, p}(\Omega)$ and $Y=L^{q}(\Omega)$, so $Y^{*}=L^{p}(\Omega)$. Analyticity of $\mathcal{G}$ and $\mathcal{E}$ has been proven in Lemmas 7.2 and 7.4, Clearly $V=W^{2, p}(\Omega) \cap W_{0}^{1, p}(\Omega) \hookrightarrow Y^{*}$ densely. Moreover, $\mathcal{E}^{\prime} \in \mathcal{C}^{\omega}\left(V ; Y^{*}\right)$ by Lemma [7.5. The Fredholm property of $\mathcal{E}^{\prime \prime}(\bar{u}): V \rightarrow Y^{*}$ has been established in Lemma 7.6. By Lemma 7.2, $\mathcal{G}^{\prime}$ extends analytically in the sense of assumption (iv) in Theorem 1.4. Moreover, the Fréchet derivative, $\mathcal{G}^{\prime \prime}(\bar{u}): V \rightarrow Y^{*}$ is compact by Lemma 7.3. By assumption, $\mathcal{G}^{\prime}(\bar{u})=g^{\prime}(\bar{u}) \not \equiv 0$, hence it is surjective as an operator $Y \rightarrow \mathbb{R}$. Thus, by Theorem 1.4, $\mathcal{M}$ is locally a manifold near $\bar{u}$ and there exist $C, \sigma>0$ and $\theta \in\left(0, \frac{1}{2}\right]$ such that for $u \in \mathcal{M}$ with $\|u-\bar{u}\|_{W^{2, p}} \leq \sigma$, we have

$$
|\mathcal{E}(u)-\mathcal{E}(\bar{u})|^{1-\theta} \leq C\left\|\mathcal{E}^{\prime}(u)\right\|_{\overline{\mathcal{T}} \boldsymbol{M}^{*}} .
$$

It remains to conclude (26) from (27). To that end, note that by Proposition 3.3 we have $\overline{\mathcal{T}_{u} \mathcal{M}}=\operatorname{ker} \overline{\mathcal{G}^{\prime}(u)}=\left\{w \in L^{q}(\Omega) \mid \int_{\Omega} g^{\prime}(u) w \mathrm{~d} x=0\right\}$. Hence, for any $\lambda \in \mathbb{R}$, we have

$$
\begin{aligned}
\left\|\mathcal{E}^{\prime}(u)\right\|_{\overline{\mathcal{T}_{u} \mathcal{M}^{*}}} & =\sup _{w \in \operatorname{ker} \overline{\mathcal{G}^{\prime}(u)}} \frac{\mathcal{E}^{\prime}(u) w}{\|w\|_{L^{q}}}=\sup _{w \in \operatorname{ker}} \frac{\int_{\Omega}\left(\mathcal{E}^{\prime}(u)\right.}{} \frac{\left.\lambda g^{\prime}(u)\right) w \mathrm{~d} x}{\|w\|_{L^{q}}} \\
& \leq \sup _{w \in L^{q}(\Omega)} \frac{\int_{\Omega}\left(\mathcal{E}^{\prime}(u)-\lambda g^{\prime}(u)\right) w \mathrm{~d} x}{\|w\|_{L^{q}(\Omega)}}=\left\|\mathcal{E}^{\prime}(u)-\lambda g^{\prime}(u)\right\|_{L^{p}(\Omega)} .
\end{aligned}
$$

Choosing $\lambda:=\frac{\int_{\Omega} \mathcal{E}^{\prime}(u) g^{\prime}(u) \mathrm{d} x}{\int_{\Omega}\left(g^{\prime}(u)\right)^{2} \mathrm{~d} x}$ yields (26).

Remark 7.8. 1. If $g(x)=x-\Gamma$ for some $\Gamma>0$ in (21), the energy $\left.\mathcal{E}\right|_{\mathcal{M}}$ corresponds to the restriction of the surface area of graph $(u)$ on the set of graphs with fixed enclosed volume $\Gamma$ with the $\mathbb{R}^{d} \times\{0\}$-hyperplane. 
2. By considering the shifted energies $\tilde{\mathcal{E}}(u)=\mathcal{E}(u+\beta)$ and $\tilde{\mathcal{G}}(u)=\mathcal{G}(u+\beta)$ for $u \in V=W^{2, p}(\Omega) \cap W_{0}^{1, p}(\Omega)$ and fixed $\beta \in W^{2, p}(\Omega)$, the result can be extended to general Dirichlet boundary data.

3. Notice that in the proof of Theorem 7.7, we have some freedom in the choice of $\lambda$. Our choice is justified, since then any solution $u=u(t)$ of the equation

$$
\partial_{t} u=-\mathcal{E}^{\prime}(u)+\lambda(u) \mathcal{G}^{\prime}(u)
$$

will preserve the constraint, i.e. $\mathcal{G}(u(t))=0$ for all $t$, provided $u$ is smooth enough.

4. It is not clear, whether our choice of $\lambda$ is optimal in the sense that it minimizes the right hand side of (28). However, in the case $p=2$, so $d=1$, our choice of $\lambda$ yields the orthogonal projection (cf. Remark 5.4), and hence minimizes the right hand side of (28).

\subsection{The Allen-Cahn equation}

The following reaction-diffusion equation plays an important role in mathematical physics, modeling the process of phase separation [9],

$$
\partial_{t} u=\varepsilon \Delta u+f(u) \text { on } \Omega \times(0, T) .
$$

Here, $T, \varepsilon>0$ and $\Omega \subset \mathbb{R}^{d}$ is a domain with $\mathcal{C}^{1,1}$-boundary. Equation (29) is the $L^{2}$-gradient flow of the Ginzburg-Landau free energy

$$
\mathcal{E}_{\varepsilon}(u):=\int_{\Omega}\left(\frac{\varepsilon}{2}|\nabla u|^{2}+W(u)\right) \mathrm{d} x,
$$

where $f(u)=-W^{\prime}(u)$. The function $W$ describes some potential, a common choice is $W(s)=\frac{1}{4 \varepsilon}\left(1-s^{2}\right)^{2}$, the double well potential.

In their celebrated works [30, 31], L. Modica and S. Mortola proved that as $\varepsilon \rightarrow 0$, the energy $\mathcal{E}_{\varepsilon}$ in (30) $\Gamma$-converges to the perimeter of a suitable level set. For our result, we will consider $\varepsilon>0$ as being fixed, and therefore, we can assume $\varepsilon=1$ without loss of generality. We define $V:=W^{2,2}(\Omega) \cap W_{0}^{1,2}(\Omega)$ and write $\mathcal{E}:=\mathcal{E}_{1}$.

In this subsection, we will use Theorem 1.4 to establish a Eojasiewicz-Simon gradient inequality for the constrained energy $\left.\mathcal{E}\right|_{\mathcal{M}}$, where $\mathcal{M}=\{u \in V \mid \mathcal{G}(u)=0\}$ and

$$
\mathcal{G}(u):=\int_{\Omega} g(u) \mathrm{d} x,
$$

for a continuous function $g: \mathbb{R} \rightarrow \mathbb{R}$, cf. Section 7.1

Theorem 7.9. Let $d \leq 3, W, g \in \mathcal{C}^{\omega}(\mathbb{R} ; \mathbb{R})$. Suppose $\bar{u} \in \mathcal{M}$ is a constrained critical point of $\left.\mathcal{E}\right|_{\mathcal{M}}$ with $g^{\prime}(\bar{u}) \not \equiv 0$. Then $\mathcal{M}$ is locally a submanifold near $\bar{u}$ and $\left.\mathcal{E}\right|_{\mathcal{M}}$ satisfies a Eojasiewicz-Simon gradient inequality, i.e. there exist $C, \sigma>0$ and $\theta \in\left(0, \frac{1}{2}\right]$ such that for any $u \in \mathcal{M}$ with $\|u-\bar{u}\|_{W^{2,2}(\Omega)} \leq \sigma$ we have

$$
|\mathcal{E}(u)-\mathcal{E}(\bar{u})|^{1-\theta} \leq C\left\|-\Delta u+W^{\prime}(u)-\frac{\left\langle-\Delta u+W^{\prime}(u), g^{\prime}(u)\right\rangle_{L^{2}(\Omega)}}{\left\|g^{\prime}(u)\right\|_{L^{2}(\Omega)}^{2}} g^{\prime}(u)\right\|_{L^{2}(\Omega)} .
$$


Proof. We can use the Hilbert space version of our main result, Corollary 5.2, with $U=V=W^{2,2}(\Omega) \cap W_{0}^{1,2}(\Omega)$ and $H=L^{2}(\Omega)$. Clearly, $V \hookrightarrow H$ densely. Moreover, we have $\nabla \mathcal{E}(u)=-\Delta u+W^{\prime}(u)$. Since $W^{\prime} \in \mathcal{C}^{\omega}(\mathbb{R} ; \mathbb{R}), V \hookrightarrow \mathcal{C}(\bar{\Omega})$ as $d \leq 3$ and $\mathcal{C}(\bar{\Omega}) \hookrightarrow H$, we conclude from Theorem 7.1 that assumption (ii) in Corollary 5.2 is satisfied. For $v \in V$, we have

$$
(\nabla \mathcal{E})^{\prime}(\bar{u}) v=\left.\frac{\mathrm{d}}{\mathrm{d} t}\right|_{t=0} \nabla \mathcal{E}(\bar{u}+t v)=-\Delta v+\left.\frac{\mathrm{d}}{\mathrm{d} t}\right|_{t=0} W^{\prime}(\bar{u}+t v)=-\Delta v+W^{\prime \prime}(\bar{u}) v .
$$

Thus, using [17, Theorem 7.26], $(\nabla \mathcal{E})^{\prime}(\bar{u}): V \rightarrow H$ is a compact perturbation of the Dirichlet-Laplacian $-\Delta: V \rightarrow H$, which is an isomorphism by standard elliptic theory [17, Theorem 9.15]. Thus, $(\nabla \mathcal{E})^{\prime}(\bar{u})$ is Fredholm of index zero by Proposition 2.5. It follows from Lemma 7.2 that the $H$-gradient of $\mathcal{G}$ is given by $\nabla \mathcal{G}(u)=g^{\prime}(u)$, so assumption (iv) in Corollary 5.2 is clearly satisfied. Similar to Lemma 7.3, the Fréchet derivative $(\nabla \mathcal{G})^{\prime}(\bar{u}) v=g^{\prime \prime}(\bar{u}) v$ is compact. Moreover, the assumption $g^{\prime}(\bar{u}) \not \equiv 0$ means that assumption (vi) in Corollary 5.2 is satisfied. Thus, (31) follows from (16) and Remark 5.4.

Remark 7.10. The condition $d \leq 3$ is only needed to prove analyticity of the energy on $W^{2,2}(\Omega) \cap W_{0}^{1,2}(\Omega)$. However, like in Section 7.1 one can consider different spaces to deal with the higher dimensional cases.

\subsection{Area of surfaces of revolution with prescribed volume}

In this subsection, we will discuss an application of Theorem 1.4 to the area of a surface of revolution with prescribed boundary and prescribed inclosed volume. To that end, let $I=[a, b] \subset \mathbb{R}$ be an interval, $V:=W^{2,2}(I) \cap W_{0}^{1,2}(I), H:=L^{2}(I)$ and consider

$$
\mathcal{E}(u):=2 \pi \int_{I}(1+u) \sqrt{1+\left(u^{\prime}\right)^{2}} \mathrm{~d} x .
$$

This is the area of the surface of revolution $S$ obtained by rotating the graph of $1+u$ around the $x$-axis. Note that by requiring $u \in V$, we impose symmetric boundary conditions $1+u(a)=1+u(b)=1$. In order to ensure that $S$ is indeed a surface, we will study $\mathcal{E}$ on the set $U:=\{u \in V \mid 1+u>0$ on $I\}$. Note that $U \subset V$ is open by the Sobolev embedding $W^{2,2}(I) \hookrightarrow \mathcal{C}(\bar{I})$.

We prescribe the volume inside $S$ by some fixed value $\nu \in \mathbb{R}$, i.e. we require

$$
\mathcal{G}(u):=\pi \int_{I}(1+u)^{2} \mathrm{~d} x-\nu=0 .
$$

Unlike in Section 7.1, here we can work in the Hilbert space framework, since the embedding $W^{2,2}(I) \hookrightarrow \mathcal{C}^{1}(\bar{I})$ ensures that the energy $\mathcal{E}$ is analytic on $U$.

Lemma 7.11. The energies $\mathcal{E}$ and $\mathcal{G}$ are analytic on $U \subset V$. Moreover, for $u \in U, v \in V$ we have

$$
\mathcal{E}^{\prime}(u) v=\langle\nabla \mathcal{E}(u), v\rangle=\int_{I} 2 \pi\left[\sqrt{1+\left(u^{\prime}\right)^{2}}-(1+u)\left(\frac{u^{\prime}}{\sqrt{1+\left(u^{\prime}\right)^{2}}}\right)^{\prime}\right] v \mathrm{~d} x
$$




$$
\mathcal{G}^{\prime}(u) v=\langle\nabla \mathcal{G}(u), v\rangle=\int_{I} 2 \pi(1+u) v \mathrm{~d} x .
$$

Moreover, the maps $U \ni u \mapsto \nabla \mathcal{E}(u) \in H$ and $U \ni u \mapsto \nabla \mathcal{G}(u) \in H$ are analytic.

Proof. Similar to Lemmas 7.2, 7.4 and 7.5,

Lemma 7.12. The Fréchet derivative $(\nabla \mathcal{E})^{\prime}(u): V \rightarrow H$ is Fredholm of index zero.

Proof. Similar to Lemma 7.6, a short computation yields for $u \in U$ and $v \in V$

$$
(\nabla \mathcal{E})^{\prime}(u) v=\frac{2 \pi(1+u)}{\left(1+\left(u^{\prime}\right)^{2}\right)^{\frac{3}{2}}} v^{\prime \prime}+K v=:-A v+K v,
$$

where $K: V \rightarrow H$ only contains terms in $v$ of order 1 or lower and is hence compact by [17, Theorem 7.26]. Note that like in Lemma 7.6, $A$ is an elliptic operator in $v$ by the embeddings $V \hookrightarrow \mathcal{C}^{1}(\bar{I}) \hookrightarrow \mathcal{C}(\bar{I})$ and the requirement $u \in U$. Thus, $\mathcal{E}^{\prime \prime}(u)$ is Fredholm of index zero by Proposition 2.5 .

Lemma 7.13. The operator $(\nabla \mathcal{G})^{\prime}(u): V \rightarrow H$ is compact for all $u \in U$.

Proof. The statement follows with the same ideas as in Lemma 7.3 .

Consequently, similar to Theorems 7.7 and 7.9 we get the following result.

Theorem 7.14. Let $\mathcal{M}:=\{u \in U \mid \mathcal{G}(u)=0\}$. Suppose $\bar{u} \in \mathcal{M}$ is a constrained critical point of $\left.\mathcal{E}\right|_{\mathcal{M}}$. Then $\mathcal{M}$ is locally a submanifold near $\bar{u}$ and satisfies a Eojasiewicz-Simon gradient inequality, i.e. there exist $C, \sigma>0$ and $\theta \in\left(0, \frac{1}{2}\right]$ such that for any $u \in \mathcal{M}$ with $\|u-\bar{u}\|_{W^{2,2}(I)} \leq \sigma$ we have

$|\mathcal{E}(u)-\mathcal{E}(\bar{u})|^{1-\theta} \leq C\left\|2 \pi \sqrt{1+\left(u^{\prime}\right)^{2}}-2 \pi(1+u)\left(\frac{u^{\prime}}{\sqrt{1+\left(u^{\prime}\right)^{2}}}\right)^{\prime}-\lambda(u) 2 \pi(1+u)\right\|_{L^{2}(I)}$,

where the scalar $\lambda(u)$ is given by

$$
\lambda(u)=\frac{3}{2 \nu} \mathcal{E}(u)-\left.\frac{\pi}{\nu} \frac{u^{\prime}}{\sqrt{1+\left(u^{\prime}\right)^{2}}}\right|_{a} ^{b}-\frac{2 \pi}{\nu} \int_{I} \frac{1+u}{\sqrt{1+\left(u^{\prime}\right)^{2}}} \mathrm{~d} x .
$$

Proof. We use Corollary 5.2, It remains to check condition (vi). Clearly, $\mathcal{G}(\bar{u})=0$ and we have $\nabla \mathcal{G}(\bar{u})=2 \pi(1+\bar{u}) \not \equiv 0 \in H=L^{2}(I)$, since $\bar{u} \in U$. Thus $\left.\mathcal{E}\right|_{\mathcal{M}}$ satisfies a Łojasiewicz-Simon gradient inequality at $\bar{u}$. By Remark 5.4, we get

$$
|\mathcal{E}(u)-\mathcal{E}(\bar{u})|^{1-\theta} \leq C\left\|\nabla \mathcal{E}(u)-\frac{\langle\nabla \mathcal{G}(u), \nabla \mathcal{E}(u)\rangle}{\|\nabla \mathcal{G}(u)\|_{L^{2}(I)}^{2}} \nabla \mathcal{G}(u)\right\|_{L^{2}(I)} .
$$

Using (32) and (33) yields (34) with $\lambda(u)=\frac{\langle\nabla \mathcal{E}(u), \nabla \mathcal{G}(u)\rangle}{\|\nabla \mathcal{G}(u)\|_{L^{2}(I)}^{2}}$. The explicit formula (35) for the scalar $\lambda$ can be proven using integration by parts and $\mathcal{G}(u)=0$. 


\section{A. Proof of Theorem 1.2}

Following the notation in [10, Section 3], let $V$ be a Banach space, $\mathcal{E} \in \mathcal{C}^{2}(U ; \mathbb{R})$ with $U \subset V$ open and let $\varphi \in U$ be a critical point of $\mathcal{E}$. Then $M:=\mathcal{E}^{\prime} \in \mathcal{C}^{1}\left(U ; V^{*}\right)$ and $L:=\mathcal{E}^{\prime \prime} \in \mathcal{C}\left(U ; \mathcal{L}\left(V, V^{*}\right)\right)$. In [10], the Łojasiewicz-Simon gradient inequality is proven under the following assumptions.

Hypothesis A.1 (Hypothesis 3.2. in [10]). The kernel $V_{0}:=\operatorname{ker} L(\varphi)$ is complemented, i.e. there exists a projection $Q \in \mathcal{L}(V)$ such that $\operatorname{Im} Q=V_{0}$.

Clearly, under the assumption of Hypothesis A.1, we have $V=V_{0} \oplus V_{1}$ with $V_{1}=\operatorname{ker} Q$, and similarly $V^{*}=V_{0}^{*} \oplus V_{1}^{*}$ with $V_{0}^{*}=\operatorname{Im} Q^{*}$ and $V_{1}^{*}=\operatorname{ker} Q^{*}$. Note that this abuse of notation is justified since $V_{1}^{*}=\left\{v^{*} \in V^{*} \mid v^{*}(w)=0\right.$ for all $\left.w \in V_{0}\right\}$ is isomorphic to the dual of $V_{0}$ and similarly for $V_{0}^{*}$.

Hypothesis A.2 (Hypothesis 3.4. in [10]). There exists a Banach space $W$ with the following properties.

(i) $W \hookrightarrow V^{*}$ continuously,

(ii) the adjoint $Q^{*} \in \mathcal{L}\left(V^{*}\right)$ of the projection in Hypothesis A.1 leaves $W$ invariant,

(iii) $M \in \mathcal{C}^{1}(U ; W)$,

(iv) $\operatorname{Im} L(\varphi)=V_{1}^{*} \cap W$.

Theorem A.3 (Corollary 3.11 in [10]). Suppose $\varphi \in U$ is a critical point of $\mathcal{E}$ and assume Hypotheses A.1 and A.2 hold. Assume that there exists Banach spaces $X \subset V$ and $Y \subset W$ such that

(i) the spaces $X$ and $Y$ are invariant under $Q$ and $Q^{*}$, respectively,

(ii) the restriction of the derivative $M$ to $U \cap X$ is analytic in a neighborhood of $\varphi$ with values in $Y$,

(iii) $\operatorname{ker} L(\varphi)$ is contained in $X$ and finite-dimensional,

(iv) $\left.\operatorname{Im} L(\varphi)\right|_{X}=\operatorname{ker} Q^{*} \cap Y$.

Then $\mathcal{E}$ satisfies the Eojasiewicz-Simon gradient inequality at $\varphi$.

Proof of Theorem 1.2. We will show that Hypotheses A.1 and A.2 and the assumptions of Theorem A.3 are satisfied for $\varphi:=\bar{u}$. We follow [13, Appendix A], where the result was proven for a special case. Let $Z$ as in Theorem [1.2, then (i) in Hypothesis A.2 is satisfied for $W:=Z^{*}$ since $V \hookrightarrow Z$ densely.

We set $X:=V, Y:=W=Z^{*}$. Since $L(\varphi)=\mathcal{E}^{\prime \prime}(\bar{u}): V \rightarrow Z^{*}$ is Fredholm by assumption (iii), its kernel $V_{0}:=\operatorname{ker} L(\varphi) \subset V \subset Z$ is finite-dimensional, thus (iii) in Theorem A.3 holds. Let $d:=\operatorname{dim} V_{0}<\infty$ and note that $V_{0}$ is closed in both $V$ and $Z$ and thus complemented in both spaces (cf. Lemma 2.8). This implies that there exists $V_{1} \subset V$ closed 
such that $V=V_{0} \oplus V_{1}$ and there exists a projection $\tilde{Q} \in \mathcal{L}(Z)$ onto $V_{0}$. We will extend this to obtain a particular projection on $Z$ onto $V_{0}$. Note that $Q:=\left.\tilde{Q}\right|_{V}: V \rightarrow V$ is also continuous, since as $V_{0}=\operatorname{Im} \tilde{Q}=\operatorname{Im} Q$ is finite-dimensional, there exist $C, C^{\prime}, C^{\prime \prime}>0$ such that $\|Q v\|_{V} \leq C\|\tilde{Q} v\|_{Z} \leq C^{\prime}\|v\|_{Z} \leq C^{\prime \prime}\|v\|_{V}$ for all $v \in V$, using that $V \hookrightarrow Z$. Now, $Q \in \mathcal{L}(V)$ satisfies Hypothesis A.1. Denote by $Q^{*} \in \mathcal{L}\left(V^{*}\right)$ the adjoint of $Q$.

Assumption (ii) in Theorem 1.2 immediately implies that assumption (iii) in Hypothesis A.2 and (ii) in Theorem A.3 are satisfied.

In order to prove Hypothesis A.2 (iv), recall that by Schwarz's Theorem (cf. [24, XIII, Theorem 5.3]), the second derivative $L(\varphi): V \rightarrow Z^{*}$ is symmetric, i.e. for $v, w \in V$ we have $(L(\varphi) v)(w)=(L(\varphi) w)(v)$. Thus, for $v \in V$ and for any $w \in V_{0}=\operatorname{ker} L(\varphi)$, we have $(L(\varphi) v)(w)=0$, i.e.

$$
\operatorname{Im} L(\varphi) \subset\left\{z^{*} \in Z^{*} \mid z^{*}(v)=0 \text { for all } v \in V_{0}\right\}=V_{1}^{*} \cap Z^{*},
$$

using $V^{*}=V_{0}^{*} \oplus V_{1}^{*}$. As a next step, we show that the inclusion in (36) is an equality. Since ind $L(\varphi)=0$, we have $\operatorname{codim}\left(\operatorname{Im} L(\varphi), Z^{*}\right)=\operatorname{dim} \operatorname{ker} L(\varphi)=\operatorname{dim} V_{0}=d$. Moreover, we have $\operatorname{codim}\left(V_{1}^{*} \cap Z^{*}, Z^{*}\right)=d$. Indeed, considering $V_{0}$ as a subspace of $Z$, by (36), we have $V_{1}^{*} \cap Z^{*}=V_{0}^{\perp}$, the annihilator of $V_{0}$ in $Z^{*}$. By [8, Proposition 11.13], we have $d=\operatorname{dim} V_{0}=\operatorname{codim} V_{0}^{\perp}=\operatorname{codim}\left(V_{1}^{*} \cap Z^{*}, Z^{*}\right)$. Hence, $\operatorname{Im} L(\varphi)$ and $V_{1}^{*} \cap Z^{*}$ are two subspaces with the same finite codimension $d$ in $Z^{*}$, with one contained in the other. Therefore, they have to be equal by [7, Proposition 5 in II $\S 7$. 3.]. Thus, equality holds in (36), so Hypothesis A.2 (iv) and assumption (iv) in Theorem A.3 are satisfied. It remains to check that $Q^{*}$ leaves $W=Z^{*}$ invariant. Let $z^{*} \in Z^{*}$ and $v \in Z$. Then $\left(Q^{*} z^{*}\right) v=z^{*}(Q v)$ is linear in $v$ and for $C=\left\|z^{*}\right\|_{Z^{*}}\|\tilde{Q}\|_{\mathcal{L}(Z)} \geq 0$, we have

$$
\left|z^{*}(Q v)\right| \leq\left\|z^{*}\right\|_{Z^{*}}\|Q v\|_{Z}=\left\|z^{*}\right\|_{Z^{*}}\|\tilde{Q} v\|_{Z} \leq C\|v\|_{Z} .
$$

By (37), $Q^{*} z^{*}: Z \rightarrow \mathbb{R}$ is an element of the dual space $Z^{*}$. This yields that Hypothesis A.2 (ii) and assumption (i) in Theorem A.3 are satisfied. Hence, we may apply Theorem $\mathrm{A} .3$ to conclude that $\mathcal{E}$ satisfies a Łojasiewicz-Simon gradient inequality in a neighborhood of $\varphi=\bar{u}$.

\section{B. Basic sequences in Banach spaces}

The goal of this subsection is to provide a proof of Theorem 6.1. We therefore use a generalization of the notion of an orthonormal basis in a Hilbert space to the Banach space situation. We follow the presentation of [3, Chapter 1].

Definition B.1 (Cf. [3, Definition 1.1.2 and Definition 1.1.5]). Let $X$ be a Banach space and $\left(e_{n}\right)_{n \in \mathbb{N}} \subset X$ be a sequence. Suppose there exists a sequence $\left(\phi_{k}\right)_{k \in \mathbb{N}} \subset X^{*}$ such that

(i) $\phi_{k}\left(e_{n}\right)=\delta_{k, n}$ for all $k, j \in \mathbb{N}$,

(ii) $v=\sum_{k=1}^{\infty} \phi_{k}(v) e_{k}$ for all $v \in X$. 
Then, $\left(e_{n}\right)_{n \in \mathbb{N}}$ is called a Schauder basis for $X$ with associated biorthogonal functionals $\left(\phi_{k}\right)_{k \in \mathbb{N}}$. A sequence $\left(e_{n}\right)_{n \in N}$ in a Banach space $X$ is called a basic sequence if it is a Schauder basis for $\overline{\operatorname{span}}\left\{e_{n} \mid n \in \mathbb{N}\right\}$.

The following is an immediate consequence of the Uniform Boundedness Principle.

Proposition B.2 (Cf. [3, Proposition 1.1.4]). Let $\left(e_{n}\right)_{n \in N}$ be a Schauder basis for a Banach space $X$. Then the natural projections $S_{N}: X \rightarrow X, S_{N} v:=\sum_{k=1}^{N} \phi_{k}(v) e_{k}$ are uniformly bounded in $\mathcal{L}(X)$, i.e. we have

$$
K:=\sup _{N \in \mathbb{N}}\left\|S_{N}\right\|<\infty .
$$

The number $K$ is called the basis constant of the sequence $\left(e_{n}\right)_{n \in \mathbb{N}}$. The following existence result is what we need to prove Theorem 6.1.

Theorem B.3. Let $V$ be an infinite-dimensional Banach space and let $\varepsilon>0$. Then there exists a basic sequence $\left(e_{n}\right)_{n \in \mathbb{N}}$ with basis constant $K \leq 1+\varepsilon$ and $\left\|e_{n}\right\|=1$ for all $n \in \mathbb{N}$.

Proof. The existence of a basic sequence $\left(e_{n}\right)_{n \in \mathbb{N}}$ with basis constant less than $1+\varepsilon$ is exactly the statement of [3, Corollary 1.5.3]. Investigating its proof, we note that the $e_{n}$ are chosen from $S:=\{x \in X \mid\|x\|=1\}$, which proves the claim.

Now, we are finally able to prove Theorem 6.1 .

Proof of Theorem 6.1. Using Theorem B.3, we obtain a sequence $\left(e_{n}\right)_{n \in \mathbb{N}}$ with $\left\|e_{n}\right\|=1$ for all $n \in \mathbb{N}$ which is a basic sequence, i.e. a Schauder basis for the Banach space $X:=\overline{\operatorname{span}}\left\{e_{n} \mid n \in \mathbb{N}\right\} \subset V$. By Definition B.1 there exists an associated biorthogonal sequence $\left(\phi_{k}\right)_{k \in \mathbb{N}} \subset X^{*}$. Note that by Theorem B.3, the basis constant $K$ defined in (38) satisfies $K \leq 1+\varepsilon$. For $x \in X$ and $k \in \mathbb{N}$, we have setting $S_{0}:=0 \in \mathcal{L}(X)$

$$
\left|\phi_{k}(x)\right|=\left\|\phi_{k}(x) e_{k}\right\|=\left\|S_{k} x-S_{k-1} x\right\| \leq 2 K\|x\|,
$$

thus $\left\|\phi_{k}\right\|_{X^{*}} \leq 2 K \leq 2(1+\varepsilon)$. By the Hahn-Banach Theorem, there exist extensions of $\phi_{k}: X \rightarrow \mathbb{R}$ also denoted $\phi_{k}: V \rightarrow \mathbb{R}$ with $\left\|\phi_{k}\right\|_{V^{*}}=\left\|\phi_{k}\right\|_{X^{*}} \leq 2(1+\varepsilon)$.

\section{Acknowledgments}

The author would like to thank Anna Dall'Acqua, Marius Müller and Adrian Spener for helpful discussions and comments.

\section{Funding}

This work was supported by the Deutsche Forschungsgemeinschaft (DFG, German Research Foundation)-Projektnummer: 404870139. 


\section{References}

[1] R. Abraham, J. E. Marsden, and T. S. Ratiu. Manifolds, tensor analysis, and applications. Springer, New York, 2004.

[2] P.-A. Absil, R. Mahony, and B. Andrews. Convergence of the iterates of descent methods for analytic cost functions. SIAM J. Optim., 16(2):531-547, 2005.

[3] F. Albiac and N. J. Kalton. Topics in Banach space theory, volume 233 of Graduate Texts in Mathematics. Springer, New York, 2006.

[4] J. Appell and M. Väth. Elemente der Funktionalanalysis. Vieweg, Wiesbaden, 2005.

[5] J. Appell and P. P. Zabrejko. Nonlinear superposition operators, volume 95 of Cambridge Tracts in Mathematics. Cambridge University Press, Cambridge, 1990.

[6] H. Attouch, J. Bolte, P. Redont, and A. Soubeyran. Proximal alternating minimization and projection methods for nonconvex problems: an approach based on the Kurdyka-Łojasiewicz inequality. Math. Oper. Res., 35(2):438-457, 2010.

[7] N. Bourbaki. Algebra I. Chapters 1-3. Elements of Mathematics (Berlin). SpringerVerlag, Berlin, 1998.

[8] H. Brezis. Functional Analysis, Sobolev Spaces and Partial Differential Equations. Springer, New York, 2010.

[9] J. W. Cahn and J. E. Hilliard. Free energy of a nonuniform system. I. Interfacial free energy. J. Chem. Phys., 28(2):258-267, 1958.

[10] R. Chill. On the Eojasiewicz-Simon gradient inequality. J. Funct. Anal., 201(2):572601, 2003.

[11] R. Chill and E. Fašangová. Gradient systems. MatFyzPress, Prague, 2010. Lecture Notes of the 13th International Internet Seminar.

[12] R. Chill, E. Fašangová, and R. Schätzle. Willmore blowups are never compact. Duke Math. J., 147(2):345-376, 2009.

[13] A. Dall'Acqua, P. Pozzi, and A. Spener. The Łojasiewicz-Simon gradient inequality for open elastic curves. J. Differential Equations, 261(3):2168-2209, 2016.

[14] P. M. N. Feehan and M. Maridakis. Lojasiewicz-Simon gradient inequalities for analytic and Morse-Bott functionals on Banach spaces and applications to harmonic maps. arXiv e-prints, page arXiv:1510.03817, 2015.

[15] E. Feireisl and F. Simondon. Convergence for semilinear degenerate parabolic equations in several space dimensions. J. Dynam. Differential Equations, 12(3):647-673, 2000 . 
[16] M. Giaquinta and S. Hildebrandt. Calculus of variations. I, volume 310 of Grundlehren der Mathematischen Wissenschaften. Springer-Verlag, Berlin, 1996.

[17] D. Gilbarg and N. S. Trudinger. Elliptic partial differential equations of second order. Classics in Mathematics. Springer, Berlin, 2001.

[18] A. Haraux and M. A. Jendoubi. Convergence of bounded weak solutions of the wave equation with dissipation and analytic nonlinearity. Calc. Var. Partial Differential Equations, 9(2):95-124, 1999.

[19] A. Haraux and M. A. Jendoubi. The Lojasiewicz gradient inequality in the infinitedimensional Hilbert space framework. J. Funct. Anal, 260(9):2826-2842, 2011.

[20] M. A. Jendoubi. Convergence of global and bounded solutions of the wave equation with linear dissipation and analytic nonlinearity. J. Differential Equations, 144(2):302-312, 1998.

[21] M. A. Jendoubi. A simple unified approach to some convergence theorems of L. Simon. J. Funct. Anal., 153(1):187-202, 1998.

[22] K. Kurdyka. On gradients of functions definable in o-minimal structures. Ann. Inst. Fourier (Grenoble), 48(3):769-783, 1998.

[23] C. Lageman. Pointwise convergence of gradient-like systems. Math. Nachr., 280(1314):1543-1558, 2007.

[24] S. Lang. Real and Functional Analysis. Springer, New York, 2012.

[25] D. Lengeler. Asymptotic stability of local Helfrich minimizers. Interfaces Free Bound., 20(4):533-550, 2018.

[26] J. Lindenstrauss and L. Tzafriri. On the complemented subspaces problem. Israel J. Math., 9:263-269, 1971.

[27] S. Łojasiewicz. Une propriété topologique des sous-ensembles analytiques réels. In Les Équations aux Dérivées Partielles (Paris), pages 87-89. Éditions du Centre National de la Recherche Scientifique, Paris, 1963.

[28] S. Łojasiewicz. Sur les ensembles semi-analytiques. I.H.E.S., Bures-sur-Yvette, 1965.

[29] S. Łojasiewicz. Sur les trajectoires du gradient d'une fonction analytique. In Geometry seminars, 1982-1983, pages 115-117. Univ. Stud. Bologna, Bologna, 1984.

[30] L. Modica. The gradient theory of phase transitions and the minimal interface criterion. Arch. Rational Mech. Anal., 98(2), 1987.

[31] L. Modica and S. Mortola. Un esempio di $\Gamma^{-}$-convergenza. Boll. Un. Mat. Ital. B (5), 14(1):285-299, 1977. 
[32] P. Rybka and K.-H. Hoffmann. Convergence of solutions to the equation of quasistatic approximation of viscoelasticity with capillarity. J. Math. Anal. Appl., 226(1):61-81, 1998.

[33] L. Simon. Asymptotics for a class of nonlinear evolution equations, with applications to geometric problems. Ann. of Math. (2), 118(3):525-571, 1983.

[34] E. F. Whittlesey. Analytic functions in Banach spaces. Proceedings of the American Mathematical Society, 16(5):1077-1083, 1965.

[35] E. Zeidler. Nonlinear Functional Analysis and its Applications: I Fixed-Point Theorems. Springer, New York, 1986. 